\title{
Inexact Implementation Using Krylov Subspace Methods for Large Scale Exponential Discriminant Analysis with Applications to High Dimensionality Reduction Problems
}

\author{
Gang Wu, Ting-ting Feng, Li-jia Zhang, Meng Yang
}

\begin{abstract}
Exponential discriminant analysis (EDA) is a generalized discriminant analysis method based on matrix exponential. It can essentially overcome the intrinsic difficulty of small sample size problem that exists in the classical linear discriminant analysis (LDA). However, for data with high dimensionality, one has to solve a large matrix exponential eigenproblem in this method, and the time complexity is dominated by the computation of exponential of large matrices. In this paper, we propose two inexact Krylov subspace algorithms for solving the large matrix exponential eigenproblem efficiently. The contribution of this work is threefold. First, we consider how to compute matrix exponential-vector products efficiently, which is the key step in the Krylov subspace method. Second, we compare the discriminant analysis criterion of EDA and that of LDA from a theoretical point of view. Third, we establish the relationship between the accuracy of the approximate eigenvectors and the distance to nearest neighbour classifier, and show why the matrix exponential eigenproblem can be solved approximately in practice. Numerical experiments on some real-world databases show the superiority of our new algorithms over their original counterpart for face recognition.
\end{abstract}

\section{Index Terms}

Dimensionality reduction, Linear discriminant analysis (LDA), Exponential discriminant analysis (EDA), Matrix exponential, Krylov subspace method.

\section{INTRODUCTION}

Dimensionality reduction is the process of reducing the number of random variables under consideration, via obtaining a set uncorrelated principle variables, which can be divided into feature selection and feature extraction. The advantages of dimensionality reduction include that it reduces the time and storage space required, improves the performance of the machine learning model, and it becomes easier to visualize the data when reduced to very low dimensions. In essence, dimensionality reduction is the transformation of high-dimensional data into a lower dimensional data space. Currently, one of the most extensively used dimensionality reduction methods is subspace transformation [10], [23], [24], [27], [40], [41], [43].

Corresponding author. G. Wu is with Department of Mathematics, China University of Mining and Technology, Xuzhou, 221116, Jiangsu, P.R. China \& School of Mathematics and Statistics, Jiangsu Normal University, Xuzhou, 221116, Jiangsu, P.R. China. E-mail: gangwu76@126. com and gangwu@cumt. edu. cn. This author is supported by the National Science Foundation of China under grant 11371176, the Natural Science Foundation of Jiangsu Province under grant BK20131126, and the Talent Introduction Program of China University of Mining and Technology.

T. Feng is with Department of Mathematics, Shanghai Key Laboratory of Pure Mathematics and Mathematical Practice, East China Normal University, Dongchuan RD 500, Shanghai, 200241, P.R. China. E-mail: tofengtingting@163.com.

L. Zhang is with School of Mathematics and Statistics, Jiangsu Normal University, Xuzhou, 221116, Jiangsu, P.R. China. E-mail: zhanglijia860163.com.

M. Yang is with School of Computer Science and Technology, Soochow University, Suzhou, 215006, Jiangsu, P.R. China. E-mail: eyangmeng@163. com. 
Linear discriminant analysis (LDA) is one of notable subspace transformation methods for dimensionality reduction [10], [12], [28]. LDA encodes discriminant information by maximizing the between-class scatter, and meanwhile minimizing the within-class scatter in the projected subspace. More precisely, let $X=\left[\mathbf{a}_{1}, \mathbf{a}_{2}, \ldots, \mathbf{a}_{n}\right]$ be a set of training samples in a $d$-dimensional feature space, and assume that the original data is partitioned into $K$ classes as $X=\left[X_{1}, X_{2}, \ldots, X_{K}\right]$. We denote by $n_{j}$ the number of samples in the $j$-th class, and thus $\sum_{j=1}^{K} n_{j}=n$. Let $\mathbf{c}_{j}$ be the centroid of the $j$-th class, and $\mathbf{c}$ be the global centroid of the training data set. If we denote $\mathbf{e}_{j}=[1,1, \ldots, 1]^{T} \in \mathbb{R}^{n_{j}}$, then the within-class scatter matrix is defined as

$$
S_{W}=\sum_{j=1}^{K} \sum_{\mathbf{a}_{i} \in X_{j}}\left(\mathbf{a}_{i}-\mathbf{c}_{j}\right)\left(\mathbf{a}_{i}-\mathbf{c}_{j}\right)^{T}=H_{W} H_{W}^{T},
$$

where $H_{W}=\left[X_{1}-\mathbf{c}_{1} \mathbf{e}_{1}^{T}, \ldots, X_{K}-\mathbf{c}_{K} \mathbf{e}_{K}^{T}\right] \in \mathbb{R}^{d \times n}$. The between-class scatter matrix is defined as

$$
S_{B}=\sum_{j=1}^{K} n_{j}\left(\mathbf{c}_{j}-\mathbf{c}\right)\left(\mathbf{c}_{j}-\mathbf{c}\right)^{T}=H_{B} H_{B}^{T},
$$

where $H_{B}=\left[\sqrt{n_{1}}\left(\mathbf{c}_{1}-\mathbf{c}\right), \sqrt{n_{2}}\left(\mathbf{c}_{2}-\mathbf{c}\right), \ldots, \sqrt{n_{K}}\left(\mathbf{c}_{K}-\mathbf{c}\right)\right] \in \mathbb{R}^{d \times K}$. The LDA method is realized by maximizing the between-class scatter distance while minimizing the within-class scatter distance, which involves solving a problem called "Ratio Trace" problem of the following form [17], [25], [28]

$$
J_{L D A}=\max _{\substack{V \in \mathcal{R}^{d \times t} \\ V^{T} V=I}} \operatorname{tr}\left(\left(V^{T} S_{W} V\right)^{-1}\left(V^{T} S_{B} V\right)\right),
$$

where $\operatorname{tr}(\cdot)$ denotes the trace of a matrix, and $t \ll d$ is the dimension of projection subspace. The optimal projection matrix $V$ can be obtained from solving the following generalized symmetric eigenproblem

$$
S_{B} \mathbf{x}=\lambda S_{W} \mathbf{x} .
$$

In practice, the dimension of real data usually exceeds the number of training samples, which results in the scatter matrix $S_{W}$ being singular. This is called the small-sample-size (SSS) or undersampled problem [28]. It is an intrinsic limitation of the classical LDA method, and it is also a common problem in classification applications [28]. In other words, the SSS problem stems from generalized eigenproblems with singular matrices. To tackle this problem, many variants of LDA have been proposed in recent years. To name a few, the regularized LDA method (RLDA) [13], LDA+PCA [4], the null-space LDA method (NLDA) [5], [6], [19], LDA/QR [48], LDA/GSVD [16], [47], the direct LDA method (DLDA) [49], the orthogonal LDA method (OLDA) [22], [46], the neighborhood minmax projections (NMMP) [26], and so on. The aforementioned variations of LDA have both advantages and disadvantages [28], [50]. An et al. [2] unified these LDA variants in one framework: principal component analysis plus constrained ridge regression.

Recently, a novel method based on matrix exponential, called exponential discriminant analysis (EDA), was proposed in [50]. Instead of the LDA criterion (1.3), EDA considers the following criterion

$$
J_{E D A}=\max _{\substack{V \in \mathcal{R}^{d \times t} \\ V^{T}=I}} \operatorname{tr}\left(\left(V^{T} \exp \left(S_{W}\right) V\right)^{-1}\left(V^{T} \exp \left(S_{B}\right) V\right)\right),
$$

where $\exp (\cdot)$ denotes exponential of a matrix. The projection matrix $V$ can be obtained from solving the $t$ dominant eigenvectors of the following generalized symmetric matrix exponential eigenproblem [50]

$$
\exp \left(S_{B}\right) \mathbf{x}=\lambda \exp \left(S_{W}\right) \mathbf{x}
$$

The framework of the EDA method for dimensionality reduction has gained wide attention in recent years. For instance, an exponential locality preserving projections (ELPP) [39] was proposed to avoid the SSS problem occured in locality preserving projections (LPP). Wang et al. [40] applied matrix exponential to extend many popular 
Laplacian embedding algorithms such as locality preserving projections, unsupervised discriminant projections, and marginal Fisher analysis. A matrix exponential local discriminant embedding method (ELDE) was investigated in [9] to deal with the SSS problem appeared in local discriminant embedding (LDE). Using the method of exponential discriminant analysis, Ahmed [1] proposed a novel image clustering model that incorporated both local and global information in image database. A 2DEDA method was presented in [45], which is an algorithm based on image matrices (2D data) rather than image vectors (1D data). Thus, 2DEDA can be viewed as a generalization of the EDA method to 2D data.

It has been widely shown that the EDA method has more discriminant power than its original counterpart [1], [9], [39], [40], [45], [50]. Indeed, in the EDA framework, the matrix exponential can be roughly interpreted as a random walk over the feature similarity matrix, and thus is more robust. As the exponentials of $S_{W}$ and $S_{B}$ are symmetric positive definite (SPD), the EDA method naturally deals with the SSS problem. Moreover, the behavior of the decay property of matrix exponential is more significant in emphasizing small distance pairs [40]. However, in all the EDA-based methods, one has to solve the large matrix exponential eigenproblem (1.6) for data with high dimensionality [1], [9], [39], [40], [45], [50], and the time complexity is dominated by the computation of $\exp \left(S_{B}\right)$ and $\exp \left(S_{W}\right)$ [50, pp.191]. This cost will be prohibitively large as the exponential of a matrix is often dense, even if the matrix in question is sparse [15]. Thus, for data with high dimension, the EDA method often suffers from heavy computational cost and storage requirement, and it is urgent to seek new schemes to speed up the solution of the large generalized matrix exponential eigenproblem arising in the framework of EDA.

Modern numerical linear algebra exploits the Krylov subspace method in different advanced iterative procedures for large scale eigenvalue problems [3], [31], [36]. Indeed, this type of method ranks among "The Top 10 Algorithms of the 20th Century" [7]. In this paper, we devote ourselves to solving (1.6) with the Krylov subspace method. The key involves the computation of matrix exponential-vector products, which is a hot topic in large scientific and engineering computations [15], [21]. In conventional approaches, one has to evaluate these products by using some iterative methods [15], [21]. In this work, we derive closed-form formulae for the matrix exponential-vector products, so that they can be formed very efficiently. The second contribution of this work is to give a theoretical comparison for the discriminant analysis criteria (1.3) and (1.5) of LDA and EDA, respectively, and show the reason why EDA can improve the classification rate of the original LDA method. Finally, we establish the relationship between the accuracy of the approximate eigenvectors and the distance to the nearest neighbour classifier (NN), and shed light on why the matrix exponential eigenproblem can be solved approximately in practice.

This paper is organized as follows. In Section 2, we briefly overview the EDA method for dimensionality reduction, and introduce two Krylov subspace methods including the Arnoldi algorithm and the Lanczos algorithm for large eigenproblems. In Section 3, we propose inexact Arnoldi and Lanczos algorithms for (1.6), and give some theoretical results to show the rationality of our new algorithms. Numerical experiments on some real-world face recognition sets including AR, Yale, Extended YaleB, FERET, and CMU-PIE are performed in Section 4. Concluding remarks are given in Section 5. Some notations used in this paper are listed in Table 1.

\section{PRELIMINARIES}

\section{A. The exponential discriminant analysis method}

Given an arbitrary square matrix $A$, its exponential is defined as [14], [15]

$$
\exp (A)=\sum_{j=0}^{\infty} \frac{A^{j}}{j !}=I+A+\frac{A^{2}}{2}+\cdots+\frac{A^{s}}{s !}+\cdots,
$$

where $I$ is the identity matrix. An important consequence is that any matrix exponential is invertible. Indeed, we have

$$
\exp (-A) \cdot \exp (A)=\exp (A-A)=I
$$

where $I$ is the identity matrix. Thus,

$$
\exp ^{-1}(A)=\exp (-A)
$$


TABLE I

Notations used in this paper

\begin{tabular}{ll}
\hline Notations & Descriptions \\
\hline$X$ & Training samples in a $d$-dimensional feature space \\
$X_{i}$ & The $i$-th class of training samples \\
$\mathbf{a}_{i}$ & The $i$-th sample \\
$d$ & Data dimension \\
$K$ & Number of classes \\
$n$ & Number of samples \\
$t$ & Dimension of projection subspace (or the number of desired eigenpairs) \\
$\ell$ & Number of samples in the training set \\
$S_{W}$ & The within-class scatter matrix defined in $(1.1)$ \\
$S_{B}$ & The between-class scatter matrix defined in $(1.2)$ \\
$\exp \left(S_{W}\right), \exp \left(S_{B}\right)$ & Exponentials of matrices $S_{W}$ and $S_{B}$ \\
$\exp$ & Square root of exp $\left(-S_{W}\right)$ \\
$e^{\mu}$ & Exponential of a scalar $\mu$ \\
$M_{N}$ & exp $\left(-S_{W}\right)$ exp $\left(S_{B}\right)$ \\
$M_{S}$ & exp ${ }^{1 / 2}\left(-S_{W}\right) \exp \left(S_{B}\right)$ exp ${ }^{1 / 2}\left(-S_{W}\right)$ \\
$\lambda, \mathbf{x}$ & Eigenvalue and eigenvector \\
$\mathcal{K}_{m}\left(A, \mathbf{v}_{1}\right)$ & Krylov subspace with respect to $A$ and $\mathbf{v}_{1}$ \\
$\operatorname{tr}(A)$ & Trace of the matrix $A$ \\
$V$ & Projection matrix composed of the desired eigenvectors \\
$A^{T}, A^{H}$ & Transpose and conjugate transpose of $A$ \\
$I$ & The identity matrix \\
$\mathbf{0}$ & Zero matrix or vector \\
$\operatorname{span}\{W\}$ & Subspace spanned by the columns of $W$ \\
$\|\cdot\|_{2}$ & 2-norm of a vector or matrix \\
\hline
\end{tabular}

Suppose that $A \in \mathbb{R}^{d \times d}$ is symmetric and let $A=X \Lambda X^{-1}$ be the eigen-decomposition of $A$, where $\Lambda=$ $\operatorname{diag}\left\{\lambda_{1}, \lambda_{2}, \ldots, \lambda_{d}\right\}$ is diagonal and $X$ is a $d \times d$ matrix with its columns being the eigenvectors. Then it is easy to see that

$$
\exp (A)=X \exp (\Lambda) X^{-1}
$$

In other words, $A$ and $\exp (A)$ share the same eigenvectors, and the eigenvalues of $\exp (A)$ are $\exp (\Lambda)=$ $\operatorname{diag}\left\{e^{\lambda_{1}}, e^{\lambda_{2}}, \ldots, e^{\lambda_{d}}\right\}$.

The exponential discriminant analysis method (EDA) makes use of the exponential criterion (1.5), and the projection matrix $V$ is obtained from solving the matrix exponential eigenproblem (1.6). This method behaves as if transforming the original data into a new space by distance diffusion mapping, and the LDA criterion is applied in such a new space [50]. The EDA method is listed as follows:

\section{Algorithm 1. The exponential discriminant analysis method (EDA) [50]}

Input: The data matrix $X=\left[\mathbf{a}_{1}, \mathbf{a}_{2}, \ldots, \mathbf{a}_{n}\right] \in \mathbb{R}^{d \times n}$, where $\mathbf{a}_{j}$ represernts the $j$-th training image.

Output: The projection matrix $V$.

1. Compute the matrices $S_{B}, S_{W}$, $\exp \left(S_{B}\right)$, and $\exp \left(S_{W}\right)$;

2. Compute the eigenvectors $\left\{\mathbf{x}_{i}\right\}$ and eigenvalues $\left\{\lambda_{i}\right\}$ of $\exp \left(S_{W}\right)^{-1} \exp \left(S_{B}\right)$;

3. Sort the eigenvectors $V=\left\{\mathbf{x}_{i}\right\}$ according to $\lambda_{i}$ in decreasing order;

4. Orthogonalize the columns of the projection matrix $V$.

As a result of diffusion mapping, the margin between different classes is enlarged in the EDA framework, which is helpful in improving classification accuracy [50]. In the small-sample-size case, EDA can extract not only the 
most discriminant information that is contained in the null space of the within-class scatter matrix, where it is similar to NLDA; but also the discriminant information that is contained in the non-null space of the within-class scatter matrix, and it is equivalent to LDA+PCA [50]. In addition, there are at most $K-1$ nonzero generalized eigenvalues in LDA, where $K$ is the number of classes in the data set, so the dimension of the projected subspace is at most $K-1$ in LDA. As a comparison, there is no (theoretical) dimensionality limitation in EDA, and we can project the input data into a low-dimensional subspace whose dimension is larger than the number of classes.

We next illustrate the superiority of the EDA method over the LDA method via a toy example; for more details, refer to [50].

Example 1.1 Consider the set of training samples $X=\left[\mathbf{a}_{1}, \mathbf{a}_{2}, \mathbf{a}_{3}\right] \in \mathbb{R}^{3 \times 3}$, whose dimension is equal to the number of samples. Let the original data be partitioned into $K=3$ classes: $X=\left[\mathbf{a}_{1}, \mathbf{a}_{2}, \mathbf{a}_{3}\right]=\left[X_{1}, X_{2}, X_{3}\right]$, and assume that the columns of $X$ are linear independent. So we have $\mathbf{c}_{i}=\mathbf{a}_{i}(i=1,2,3)$, the global centroid $\mathbf{c}=\left(\mathbf{c}_{1}+\mathbf{c}_{2}+\mathbf{c}_{3}\right) / 3$, and

$$
\begin{gathered}
H_{W}=\left[\mathbf{a}_{1}-\mathbf{c}_{1}, \mathbf{a}_{2}-\mathbf{c}_{2}, \mathbf{a}_{3}-\mathbf{c}_{3}\right]=\mathbf{0}, \\
H_{B}=\left[\mathbf{a}_{1}-\mathbf{c}, \mathbf{a}_{2}-\mathbf{c}, \mathbf{a}_{3}-\mathbf{c}\right] .
\end{gathered}
$$

Therefore, $S_{W}=H_{W} H_{W}^{T}=\mathbf{0}, S_{B}=H_{B} H_{B}^{T}$, and the generalized eigenproblem (1.4) for the LDA method turns out to be

$$
S_{B} \mathbf{x}=\mathbf{0} .
$$

Since the columns of $X$ are linear independent, we have $\operatorname{rank}\left(S_{B}\right)=2$, and there is only one solution vector (up to a scalar) for (2.4).

As a comparison, the matrix-exponential eigenproblem (1.6) for the EDA method reduces to

$$
\exp \left(S_{B}\right) \mathbf{x}=\lambda \mathbf{x},
$$

which has three linear independent solution vectors. Therefore, the LDA method loses some useful discriminant information, and the EDA method can perform better than the LDA method.

As was stressed in [50, pp.191], the time complexity of EDA is dominated by the computation of $\exp \left(S_{B}\right)$ and $\exp \left(S_{W}\right)$, as well as the evaluation of the large matrix exponential eigenproblem. More precisely, one has to explicitly form and store $\exp \left(S_{B}\right)$ and $\exp \left(-S_{W}\right)$, as well as their product $\exp \left(-S_{W}\right) \exp \left(S_{B}\right)$. The complexity is $\mathcal{O}\left(d^{3}\right)$, which is prohibitively large as the dimension of the data is high [15]. Moreover, we have to solve a large matrix exponential eigenproblem with respect to $\exp \left(S_{W}\right)^{-1} \exp \left(S_{B}\right)$. If the matrix exponential eigenproblem is solved by using the implicit QR algorithm [3], [14], another $\mathcal{O}\left(d^{3}\right)$ flops are required [3], [14]. Therefore, it is urgent to seek new strategies to improve the numerical performance of the EDA method.

\section{B. The Arnoldi algorithm and the Lanczos algorithm for large eigenproblems}

In this subsection, we briefly introduce Krylov subspace methods for large eigenproblems, in particular the Arnoldi algorithm and the Lanczos algorithm. An important class of techniques for large scale matrix computations is Krylov subspace methods, which extract approximations from the following subspace

$$
\mathcal{K}_{m}(A, \mathbf{v})=\operatorname{span}\left\{\mathbf{v}, A \mathbf{v}, \ldots, A^{m-1} \mathbf{v}\right\},
$$

called the Krylov subspace with respect to $A$ and $\mathbf{v}$ [3], [31], [36]. Here $A$ is a matrix, $\mathbf{v}$ is a vector, and $m>1$ is a positive integer.

The Arnoldi algorithm is a widely used Krylov subspace method for finding a few extreme eigenvalues and their associated eigenvectors of a large nonsymmetric matrix [3], [31], [36]. It requires only matrix-vector products to extract eigen-information to compute the desired solutions, and thus is very attractive in practice when the matrix is too large to be solved by, e.g., the implicit QR algorithm [14]; or the matrix does not exist explicitly but in the form of being capable of generating matrix-vector multiplications. 
The principle of the Arnoldi algorithm is described as follows. Given a matrix $A$ and an initial real vector $\mathbf{v}_{1}$ of unit norm, in exact arithmetics, the $m$-step Arnoldi process recursively generates an orthonormal basis $V_{m}=\left[\mathbf{v}_{1}, \mathbf{v}_{2}, \ldots, \mathbf{v}_{m}\right]$ for the Krylov subspace. At the same time, the projection of $A$ onto $\mathcal{K}_{m}\left(A, \mathbf{v}_{1}\right)$ is expressed as an upper Hessenberg matrix $H_{m}=V_{m}^{T} A V_{m}$. Afterwards the Rayleigh-Ritz procedure [3], [31], [36] is applied to compute approximate eigenpairs of $A$ : Let $(\widetilde{\lambda}, \widetilde{\mathbf{y}})$ be some eigenpairs of $H_{m}$, then we construct some approximate eigenpairs $\left(\widetilde{\lambda}, V_{m} \widetilde{\mathbf{y}}\right)$ of $A$. Here $\widetilde{\lambda}$ is called a Ritz value and $\widetilde{\mathbf{x}}=V_{m} \widetilde{\mathbf{y}}$ is called a Ritz vector of $A$ in the Krylov subspace $\mathcal{K}_{m}\left(A, \mathbf{v}_{1}\right)$. We next present a prototype of the (non-restarted) Arnoldi algorithm for evaluating $k$ dominant eigenpairs of a matrix $A$ in magnitude. For more details and practical implementations, refer to [3], [31], [36] and the references therein.

\section{Algorithm 2. [31] A prototype of the Arnoldi algorithm}

function $[\widetilde{\Lambda}, \widetilde{X}]=\operatorname{Arnoldi}\left(A, \mathbf{v}_{1}, m, k\right)$

- Run the m-step Arnoldi process:

1. for $j=1,2, \ldots, m$

2. $\mathbf{q}=A \mathbf{v}_{j}$;

3. for $i=1,2, \ldots, j$

4. $\quad h_{i, j}=\mathbf{v}_{i}^{T} \mathbf{q}$;

5. $\quad \mathbf{q}=\mathbf{q}-h_{i, j} \mathbf{v}_{i}$

6. end for

7. $h_{j+1, j}=\|\mathbf{q}\|_{2}$;

8. if $h_{j+1, j}=0$

9. break;

10. end if

11. $\mathbf{v}_{j+1}=\mathbf{q} / h_{j+1, j}$;

12. end for

- Solve the eigenproblem with respect to the $m \times m$ upper Hessenberg matrix $H_{m}=\left\{h_{i j}\right\}_{i, j=1}^{m}$. Let $\left(\widetilde{\lambda}_{i}, \widetilde{\mathbf{y}}_{i}\right), i=$ $1,2, \ldots, k$ be the $k$ dominate eigenpairs of $H_{m}$ in magnitude, we then use $\widetilde{\Lambda}=\left\{\widetilde{\lambda}_{1}, \ldots, \widetilde{\lambda}_{k}\right\}$ and $\widetilde{X}=\left[\widetilde{\mathbf{x}}_{1}, \ldots, \widetilde{\mathbf{x}}_{k}\right]$ to approximate the $k$ dominant eigenvalues of $A$ in magnitude and the corresponding eigenvectors, respectively, where $\widetilde{\mathbf{x}}_{i}=V_{m} \widetilde{\mathbf{y}}_{i}$ and $V_{m}=\left[\mathbf{v}_{1}, \mathbf{v}_{2}, \ldots, \mathbf{v}_{m}\right]$. Here $\left(\widetilde{\lambda}_{i}, \widetilde{\mathbf{x}}_{i}\right)$ is called a Ritz pair of $A$, and the corresponding residual norms are defined as $\left\|\widetilde{\mathbf{r}}_{i}\right\|_{2}=\left\|A \widetilde{\mathbf{x}}_{i}-\widetilde{\lambda}_{i} \widetilde{\mathbf{x}}_{i}\right\|_{2}, i=1,2, \ldots, k$.

The (symmetric) Lanczos algorithm can be viewed as a simplification of Arnoldi's method for the particular case when the matrix $A$ is symmetric [3], [31], [36]. The principle of the method is the same as that of the Arnoldi algorithm. However, there are a number of interesting properties that will cause the algorithm to simplify. For instance, it leads to three-term recurrences in the Lanczos procedure [3], [36], and thus the computational complexities are fewer than those of the Arnoldi algorithm. Moreover, the projection matrix $H_{m}$ is symmetric tridiagonal instead of upper Hessenberg in the Lanczos algorithm. We next give a prototype of the (non-restarted) Lanczos algorithm for calculating the $k$ dominant eigenpairs of $A$ in magnitude.

\section{Algorithm 3. [31] A prototype of the Lanczos algorithm}

function $[\widetilde{\Lambda}, \widetilde{X}]=\operatorname{Lanczos}\left(A, \mathbf{v}_{1}, m, k\right)$

- Run the m-step Lanczos process:

1. Let $\beta_{1}=0, \mathbf{v}_{0}=\mathbf{0}$;

2. for $i=1,2, \ldots, m$

3. $\mathbf{w}_{i}=A \mathbf{v}_{i}-\beta_{i} \mathbf{v}_{i-1}$;

4. $\alpha_{i}=\left(\mathbf{w}_{i}, \mathbf{v}_{i}\right)$;

5. $\mathbf{w}_{i}=\mathbf{w}_{i}-\alpha_{i} \mathbf{v}_{i}$;

6. $\beta_{i+1}=\left\|\mathbf{w}_{i}\right\|_{2}$; 


\section{7. if $\beta_{i+1}=0$ \\ 8. break; \\ 9. end if}

10. $\mathbf{v}_{i+1}=\mathbf{w}_{i} / \beta_{i+1}$;

11. end for

- Solve the eigenproblem with respect to the $m \times m$ tridiagonal matrix $H_{m}$ composed of the $\left\{\alpha_{i}\right\}_{\widetilde{\Lambda}}^{\prime} s$ and the $\left\{\beta_{i}\right\}^{\prime} s$. Let $\left(\widetilde{\lambda}_{i}, \widetilde{\mathbf{y}}_{i}\right), i=1,2, \ldots, k$ be the $k$ dominate eigenpairs of $H_{m}$ in magnitude, and then use $\widetilde{\Lambda}=\left\{\widetilde{\lambda}_{1}, \ldots, \widetilde{\lambda}_{k}\right\}$ and $\widetilde{X}=\left[\widetilde{\mathbf{x}}_{1}, \ldots, \widetilde{\mathbf{x}}_{k}\right]$ to approximate the $k$ dominant eigenvalues of $A$ in magnitude and the corresponding eigenvectors, respectively, where $\widetilde{\mathbf{x}}_{i}=V_{m} \widetilde{\mathbf{y}}_{i}$ and $V_{m}=\left[\mathbf{v}_{1}, \mathbf{v}_{2}, \ldots, \mathbf{v}_{m}\right]$. Here $\left(\widetilde{\lambda}_{i}, \widetilde{\mathbf{x}}_{i}\right)$ is called a Ritz pair of A, and the corresponding residual norms are defined as $\left\|\widetilde{\mathbf{r}}_{i}\right\|_{2}=\left\|A \widetilde{\mathbf{x}}_{i}-\widetilde{\lambda}_{i} \widetilde{\mathbf{x}}_{i}\right\|_{2}, i=1,2, \ldots, k$.

\section{KRYLOV SUBSPACE ALGORITHMS FOR MATRIX EXPONENTIAL DISCRIMINANT ANALYSIS}

In this section, we propose two Krylov subspace methods for solving (1.6), i.e., the Arnoldi algorithm and the Lanczos algorithm. We first consider how to compute the matrix exponential-vector products involved in the Krylov subspace algorithms, and then derive new lower and upper bounds on the EDA criterion (1.5) and the LDA criterion (1.3). Finally, we shed light on why the exponential eigenproblem can be solved "inexactly" in practice, and provide a practical stopping criterion for solving the matrix-exponential eigenproblem approximately. By "inexactly" we mean solving the large exponential eigenproblem (1.6) "approximately" to some given accuracy (say $10^{-4}$ ) in the exponential discriminant analysis method.

\section{A. Computing matrix exponential-vector products efficiently}

The generalized matrix exponential eigenproblem (1.6) is mathematically equivalent to the following standard nonsymmetric matrix exponential eigenproblem

$$
\exp \left(-S_{W}\right) \exp \left(S_{B}\right) \mathbf{x}=\lambda \mathbf{x}
$$

where we used $\exp ^{-1}\left(S_{W}\right)=\exp \left(-S_{W}\right)$. As it is only required to calculate $t(t \ll d)$ dominant eigenpairs of the large matrix $\exp \left(-S_{W}\right) \exp \left(S_{B}\right)$, we are interested in solving the large matrix exponential eigenproblem by using the Arnoldi algorithm.

Denote $M_{N}=\exp \left(-S_{W}\right) \exp \left(S_{B}\right)$, it is seen from Algorithm 2 that the key ingredient of the Arnoldi algorithm is to evaluate matrix exponential-vector products

$$
\mathbf{w}=M_{N} \mathbf{v}=\exp \left(-S_{W}\right) \exp \left(S_{B}\right) \mathbf{v}
$$

for different given vectors $\mathbf{v}$. When the matrices $S_{W}, S_{B}$ are large, Krylov subspace methods are widely used for this type of problem [15], [21]. Generally speaking, there are two classes of Krylov subspace methods for evaluating (3.2) iteratively [30]. In the first class of methods, the matrix is projected into a much smaller subspace, then the exponential is applied to the reduced matrix, and finally the approximation is projected back to the original large space [32]. In the second class of methods, the exponential function is first approximated by simpler functions such as rational functions, and then the action of the matrix exponential is calculated [42]. In this case, the core of (3.2) reduces to solving some linear systems with multiple shifts [44].

Thanks to the structure of $\exp \left(-S_{W}\right)$ and $\exp \left(S_{B}\right)$, instead of evaluating (3.2) iteratively, we can compute it directly by using a closed-form formula. More precisely, let

$$
H_{W}=Q_{W} R_{W}, \quad H_{B}=Q_{B} R_{B}
$$

be the (skinny) QR decomposition [14] of the matrices $H_{W}$ and $H_{B}$ from (1.1) and (1.2), respectively, where $Q_{B} \in \mathbb{R}^{d \times K}, Q_{W} \in \mathbb{R}^{d \times n}$ are orthonormal, and $R_{B} \in \mathbb{R}^{K \times K}, R_{W} \in \mathbb{R}^{n \times n}$ are upper triangular. Denote by

$$
R_{B}=U_{B} \Sigma_{B} V_{B}^{T}, \quad R_{W}=U_{W} \Sigma_{W} V_{W}^{T}
$$


the singular value decomposition (SVD) [14] of $R_{B}$ and $R_{W}$, respectively, and let

$$
\widetilde{Q}_{B}=Q_{B} U_{B}, \quad D_{B}=\Sigma_{B}^{2},
$$

and

$$
\widetilde{Q}_{W}=Q_{W} U_{W}, \quad D_{W}=\Sigma_{W}^{2},
$$

Then we have the following theorem:

Theorem 1. Let $\widetilde{Q}_{B}^{\perp}$ and $\widetilde{Q}_{W}^{\perp}$ be orthonormal bases for the orthogonal complement of $\operatorname{span}\left\{\widetilde{Q}_{B}\right\}$ and $\operatorname{span}\left\{\widetilde{Q}_{W}\right\}$, respectively, and let $f$ be a function defined on the spectra of $S_{W}$ and $S_{B}$ defined in (1.1) and (1.2), respectively.

Then

$$
f\left(S_{B}\right)=\widetilde{Q}_{B} f\left(D_{B}\right) \widetilde{Q}_{B}^{T}+\widetilde{Q}_{B}^{\perp} f\left(\mathbf{0}_{(d-K) \times(d-K)}\right)\left(\widetilde{Q}_{B}^{\perp}\right)^{T}
$$

and

$$
f\left(S_{W}\right)=\widetilde{Q}_{W} f\left(D_{W}\right) \widetilde{Q}_{W}^{T}+\widetilde{Q}_{W}^{\perp} f\left(\mathbf{0}_{(d-n) \times(d-n)}\right)\left(\widetilde{Q}_{W}^{\perp}\right)^{T},
$$

where $\mathbf{0}_{(d-K) \times(d-K)}$ and $\mathbf{0}_{(d-n) \times(d-n)}$ stand for $(d-K) \times(d-K)$ and $(d-n) \times(d-n)$ zero matrices, respectively. In particular, for matrix exponential, we have

$$
\begin{gathered}
\exp \left(S_{B}\right)=\widetilde{Q}_{B} \exp \left(D_{B}\right) \widetilde{Q}_{B}^{T}+I-\widetilde{Q}_{B} \widetilde{Q}_{B}^{T}, \\
\exp \left(-S_{W}\right)=\widetilde{Q}_{W} \exp \left(-D_{W}\right) \widetilde{Q}_{W}^{T}+I-\widetilde{Q}_{W} \widetilde{Q}_{W}^{T},
\end{gathered}
$$

and

$$
\exp ^{1 / 2}\left(-S_{W}\right)=\widetilde{Q}_{W} \exp \left(-\frac{1}{2} D_{W}\right) \widetilde{Q}_{W}^{T}+I-\widetilde{Q}_{W} \widetilde{Q}_{W}^{T},
$$

where $\exp ^{1 / 2}\left(-S_{W}\right)$ represents the square root of $\exp \left(-S_{W}\right)$.

Proof. See the Appendix.

Remark 1. We mention that the above results are independently of Krylov subspace methods. According to (3.9) and (3.10), for a given vector $\mathbf{v}$, we can update the matrix exponential-vector product (3.2) in the Arnoldi process as follows:

$\%$ To compute $M_{N} \mathbf{v}$

function $\mathbf{v}=\operatorname{UpdateArnoldi}\left(\widetilde{Q}_{B}, D_{B}, \widetilde{Q}_{W}, D_{W}, \mathbf{v}\right)$

$\mathbf{v}:=\widetilde{Q}_{B} \exp \left(D_{B}\right)\left(\widetilde{Q}_{B}^{T} \mathbf{v}\right)+\mathbf{v}-\widetilde{Q}_{B}\left(\widetilde{Q}_{B}^{T} \mathbf{v}\right)$

$\mathbf{v}:=\widetilde{Q}_{W} \exp \left(-D_{W}\right)\left(\widetilde{Q}_{W}^{T} \mathbf{v}\right)+\mathbf{v}-\widetilde{Q}_{W}\left(\widetilde{Q}_{W}^{T} \mathbf{v}\right)$.

Notice that $D_{B}$ and $D_{W}$ are $K \times K$ and $n \times n$ diagonal matrices, respectively. Therefore, there is no need to explicitly form and store the large matrices $S_{B}, S_{W}$ and their exponentials $\exp \left(S_{B}\right), \exp \left(-S_{W}\right)$ in the EDA framework.

On the other hand, recall that $S_{W}$ and $S_{B}$ and their exponentials are all symmetric matrices. Thus, it is interesting to transform (1.6) into a symmetric eigenproblem. Indeed, the generalized matrix exponential eigenproblem (1.6) can also be reformulated as a standard symmetric eigenvalue problem, say, by using $\exp \left(S_{W}\right)$ 's Cholesky decomposition [14]. Unfortunately, calculating the Cholesky decomposition of a large matrix can be very expensive. Rather than using the Cholesky decomposition, we consider $\exp \left(S_{W}\right)$ 's square root [15] that is advantageous for both computational and theoretical purposes. By (1.6),

$$
\left[\exp ^{-1 / 2}\left(S_{W}\right) \exp \left(S_{B}\right) \exp ^{-1 / 2}\left(S_{W}\right)\right]\left[\exp ^{1 / 2}\left(S_{W}\right) \mathbf{x}\right]=\lambda\left[\exp ^{1 / 2}\left(S_{W}\right) \mathbf{x}\right]
$$


Denote $\exp ^{-1 / 2}\left(S_{W}\right)$ the inverse of square root of $\exp \left(S_{W}\right)$, and note that $\exp ^{-1 / 2}\left(S_{W}\right)=\exp ^{1 / 2}\left(-S_{W}\right)$, can be rewritten as

$$
\left[\exp ^{1 / 2}\left(-S_{W}\right) \exp \left(S_{B}\right) \exp ^{1 / 2}\left(-S_{W}\right)\right] \mathbf{y}=\lambda \mathbf{y}
$$

and

$$
\mathbf{x}=\exp ^{1 / 2}\left(-S_{W}\right) \mathbf{y}
$$

is the desired solution. Notice that the matrix

$$
M_{S}=\exp ^{1 / 2}\left(-S_{W}\right) \exp \left(S_{B}\right) \exp ^{1 / 2}\left(-S_{W}\right)
$$

is a symmetric positive definite (SPD), and our aim is to compute a few dominate eigenpairs of it.

Similar to the Arnoldi algorithm, in the Lanczos algorithm shown in Algorithm 3, we need to compute the matrix exponential-vector products

$$
\mathbf{w}=M_{S} \mathbf{v}=\exp ^{1 / 2}\left(-S_{W}\right) \exp \left(S_{B}\right) \exp ^{1 / 2}\left(-S_{W}\right) \mathbf{v}
$$

for different vectors $\mathbf{v}$. In terms of (3.9), (3.10) and (3.11), for a given vector $\mathbf{v}$, we can update the matrix exponential-vector product (3.16) as follows:

$\%$ To compute $M_{S} \mathbf{v}$

function $\mathbf{v}=\operatorname{UpdateLanczos}\left(\widetilde{Q}_{B}, D_{\widetilde{Q}}, \widetilde{Q}_{W}, D_{W}, \mathbf{v}\right)$

$\mathbf{v}:=\widetilde{Q}_{W} \exp \left(-1 / 2 \times D_{W}\right) \widetilde{Q}_{W}^{T} \mathbf{v}+\mathbf{v}-\widetilde{Q}_{W} \widetilde{Q}_{W}^{T} \mathbf{v} ;$

$\mathbf{v}:=\widetilde{Q}_{B} \exp \left(D_{B}\right) \widetilde{Q}_{B}^{T} \mathbf{v}+\mathbf{v}-\widetilde{Q}_{B} \widetilde{Q}_{B}^{T} \mathbf{v}$;

$\mathbf{v}:=\widetilde{Q}_{W} \exp \left(-1 / 2 \times D_{W}\right) \widetilde{Q}_{W}^{T} \mathbf{v}+\mathbf{v}-\widetilde{Q}_{W} \widetilde{Q}_{W}^{T} \mathbf{v}$.

Compared with the Arnoldi algorithm for the standard nonsymmetric matrix exponential eigenproblem (3.1), the advantage of the Lanczos algorithm for (3.13) is that one can use three-term recurrences (i.e., $\beta_{i+1} \mathbf{v}_{i+1}=$ $A \mathbf{v}_{i}-\alpha_{i} \mathbf{v}_{i}-\beta_{i} \mathbf{v}_{i-1}$, see Steps 3-5 in Algorithm 3) in the Lanczos procedure for a symmetric eigenproblem [3], [36], instead of the long term recurrences in the Arnoldi procedure. A natural question is: which one is better, Arnoldi or Lanczos? We believe that the answer is problem-dependent. More precisely, we cannot tell which one is definitely better than the other; refer to the numerical experiments performed in Section 4. In summary, we list the pseudo-code for computing (3.2) and (3.16):

\section{Algorithm 4. Computing the matrix exponential-vector product (3.2)/(3.16) in the Arnoldi/Lanczos algorithm}

- Preprocessing:

1. Given the training set $X$, form the matrices $H_{B}$ and $H_{W}$;

2. Computing the skinny $Q R$ decompositions: $H_{B}=Q_{B} R_{B}, H_{W}=Q_{W} R_{W}$;

3. Computing SVDs: $R_{B}=U_{B} \Sigma_{B} V_{B}^{T}, R_{W}=U_{W} \Sigma_{W} V_{W}^{T}$;

4. Let $Q_{B}:=Q_{B} U_{B}, Q_{W}:=Q_{W} U_{W}, \Sigma_{B}:=\exp \left(\Sigma_{B}^{2}\right)$;

- Computing:

5. Given a vector $\mathbf{v}$;

Updating (3.2) in the Arnoldi algorithm \% UpdateArnoldi

6A. Let $\Sigma_{W}:=\exp \left(-\Sigma_{W}^{2}\right)$;

$7 A . \mathbf{w}:=Q_{B}^{T} \mathbf{v}$;

$8 A . \mathbf{v}:=Q_{B} \Sigma_{B} \mathbf{w}+\mathbf{v}-Q_{B} \mathbf{w}$

$9 A . \mathbf{w}:=Q_{W}^{T} \mathbf{v}$;

10A. $\mathbf{w}:=Q_{W} \Sigma_{W} \mathbf{w}+\mathbf{v}-Q_{W} \mathbf{w} ;$

end

Updating (3.16) in the Lanczos process \% UpdateLanczos 
6L. Let $\Sigma_{W}:=\exp \left(-1 / 2 \times \Sigma_{W}^{2}\right)$;

7L. $\mathbf{w}:=Q_{W}^{T} \mathbf{v}$;

$8 L . \mathbf{v}:=Q_{W} \Sigma_{W} \mathbf{w}+\mathbf{v}-Q_{W} \mathbf{w} ;$

$9 L . \mathbf{w}:=Q_{B}^{T} \mathbf{v}$;

10L. $\mathbf{v}:=Q_{B} \Sigma_{B} \mathbf{w}+\mathbf{v}-Q_{B} \mathbf{w} ;$

$11 L . \mathbf{w}:=Q_{W}^{T} \mathbf{v}$

$12 L . \mathbf{w}:=Q_{W} \Sigma_{W} \mathbf{w}+\mathbf{v}-Q_{W} \mathbf{w} ;$

end

\section{B. A comparison of the discriminant analysis criteria of EDA and LDA}

In this subsection, we establish new lower and upper bounds for the criteria (1.3) and (1.5), and give a theoretical comparison of the LDA method and the EDA method. Recall from (3.1) and (3.12) that, the eigenvalues of the matrix $M_{S}$ defined in (3.15) are the same as those of $M_{N}=\exp \left(-S_{W}\right) \exp \left(S_{B}\right)$ and the matrix exponential pencil $\left(\exp \left(S_{B}\right), \exp \left(S_{W}\right)\right)$. For notation simplicity, $M$ stands both for the nonsymmetric matrix $M_{N}$ and the symmetric matrix $M_{S}$ in the following theorem.

Theorem 2. Denote by $\nu_{1} \geq \nu_{2} \geq \cdots \geq \nu_{d}$ the eigenvalues of $S_{W}$, by $\mu_{1} \geq \mu_{2} \geq \cdots \geq \mu_{d}$ the eigenvalues of $S_{B}$, and let $\lambda_{1}(M) \geq \lambda_{2}(M) \geq \cdots \geq \lambda_{d}(M)$ be the eigenvalues of $M$. Then we have

$$
\max \left\{e^{\mu_{i}-\nu_{1}}, e^{\mu_{d}-\nu_{d-i+1}}\right\} \leq \lambda_{i}(M) \leq \min \left\{e^{\mu_{i}-\nu_{d}}, e^{\mu_{1}-\nu_{d-i+1}}\right\}, \quad i=1,2, \ldots, d .
$$

Proof. See the Appendix.

We are in a position to establish new lower and upper bounds for the EDA criterion (1.5). First we need a lemma.

Lemma 3. [38, Corollary 4.6.4, pp.101] Let $A$ and $B$ be two $d \times d$ Hermitian matrix, where $A$ is semi-positive definite and $B$ is positive definite. Let $X$ be a $d \times t$ matrix with $\operatorname{rank}(X)=t$. Then

$$
\operatorname{tr}\left(X^{H} A X\left(X^{H} B X\right)^{-1}\right) \geq \sum_{i=1}^{t} \lambda_{d-t+i}\left(B^{-1} A\right),
$$

and

$$
\operatorname{tr}\left(X^{H} A X\left(X^{H} B X\right)^{-1}\right) \leq \sum_{i=1}^{t} \lambda_{i}\left(B^{-1} A\right) .
$$

Combining Theorem 2 and Lemma 3, we get the following theorem for the exponential discriminant analysis criterion (1.5).

Theorem 4. Under the above notations, for the EDA criterion, there holds

$$
\sum_{i=1}^{t} \max \left\{e^{\mu_{d-t+i}-\nu_{1}}, e^{\mu_{d}-\nu_{t-i+1}}\right\} \leq J_{E D A} \leq \sum_{i=1}^{t} \min \left\{e^{\mu_{i}-\nu_{d}}, e^{\mu_{1}-\nu_{d-i+1}}\right\} .
$$

Proof. This result is from Lemma 3, (3.17), and the fact that

$$
\lambda_{d-t+i}\left(\exp \left(-S_{W}\right) \exp \left(S_{B}\right)\right) \geq \max \left\{e^{\mu_{d-t+i}-\nu_{1}}, e^{\mu_{d}-\nu_{t-i+1}}\right\}, \quad i=1,2, \ldots, d .
$$

If $S_{W}$ is nonsingular, we have the following result on the LDA criterion (1.3), whose proof is similar to that of Theorem 4, and thus is omitted. 
Theorem 5. If $S_{W}$ is nonsingular, let $\nu_{1} \geq \nu_{2} \geq \cdots \geq \nu_{d}>0, \mu_{1} \geq \mu_{2} \geq \cdots \geq \mu_{d}$ be the eigenvalues of $S_{W}$ and $S_{B}$. Then we have that

$$
\sum_{i=1}^{t} \max \left\{\frac{\mu_{d-t+i}}{\nu_{1}}, \frac{\mu_{d}}{\nu_{t-i+1}}\right\} \leq J_{L D A} \leq \sum_{i=1}^{t} \min \left\{\frac{\mu_{i}}{\nu_{d}}, \frac{\mu_{1}}{\nu_{d-i+1}}\right\} .
$$

Remark 2. It was assumed in [50, pp.189] that if

$$
\frac{e^{\mu_{i}}}{e^{\nu_{i}}}>\frac{\mu_{i}}{\nu_{i}}, \quad i=1,2, \ldots, t,
$$

then there is a difference in diffusion scale between the within-class and between-class distances, and the diffusion scale to the between-class distance is larger than the within-class distance. Hence, the margin between different classes is enlarged, which is helpful in improving classification accuracy. Theorems 4-5 show this more precisely: they indicate that if

$$
\frac{e^{\mu_{i}}}{e^{\nu_{d}}}>\frac{\mu_{i}}{\nu_{d}} \quad \text { or } \quad \frac{e^{\mu_{1}}}{e^{\nu_{d-i+1}}}>\frac{\mu_{1}}{\nu_{d-i+1}}, \quad i=1,2, \ldots, t,
$$

then $J_{E D A}$ can be larger than $J_{L D A}$. Notice that the conditions in (3.21) are (much) weaker than those in (3.20). So our result is stronger than the one given in [50], and it is useful to clarify the motivation of EDA.

\section{Solving the matrix exponential eigenproblems approximately}

In our new strategies, one needs to solve the large matrix exponential eigenproblems (3.1) or (3.12) in a prescribed accuracy. If the desired accuracy is too high (say, $\varepsilon=10^{-10}$ or even the machine precision $\epsilon \approx 2.22 \times 10^{-16}$ ), then the cost for solving the large eigenproblems will be very high. In this subsection, we give some theoretical analysis on the relationship between the accuracy of the eigenvectors and the distance measure of the nearest neighbour classifier $(\mathrm{NN})$ [8]. Based on the proposed results, we provide a practical stopping criterion for the matrix exponential eigenproblems.

$\mathrm{K}$ nearest neighbors $(\mathrm{KNN})$ is a simple algorithm that stores all available cases and classifies new cases based on a similarity measure [11], e.g., the Euclidean distance. In KNN classification, the output is a class membership. An object is classified by a majority vote of its neighbors, with the object being assigned to the class most common among its $K$ nearest neighbors (where $K$ is a positive integer, typically small). If $K=1$, then the case is simply assigned to the class of its nearest neighbor (NN) [8].

Denote by $V, \widetilde{V} \in \mathbb{R}^{d \times \ell}$ the orthonormal matrices whose columns are the "exact" and "computed" solutions of (3.1) or (3.12), respectively. Let $\widehat{\mathbf{x}}_{i}$ be a sample from the training set, and $\widehat{\mathbf{y}}_{j}$ be a sample from the testing set. Then the nearest neighbour classifier gives class membership via investigating the Euclidean distance [8]

$$
d_{i j}=\left\|V V^{T}\left(\widehat{\mathbf{x}}_{i}-\widehat{\mathbf{y}}_{j}\right)\right\|_{2}=\left\|V^{T}\left(\widehat{\mathbf{x}}_{i}-\widehat{\mathbf{y}}_{j}\right)\right\|_{2},
$$

where $\|\cdot\|_{2}$ denotes 2-norm (or the spectral norm) of a vector or matrix. Consider

$$
\sin \angle(V, \widetilde{V})=\left\|\left(I-V V^{T}\right) \widetilde{V}\right\|_{2}
$$

the distance between the eigenspace $\operatorname{span}\{V\}$ and the approximate eigenspace $\operatorname{span}\{\widetilde{V}\}$ [36]. Notice that $\sin \angle(V, \widetilde{V})$ uses the spectral norm and that it equals the greatest singular value of $\widetilde{V}$ projected onto the orthogonal complement of $V$, moreover, $0 \leq \sin \angle(V, \widetilde{V}) \leq 1$ and $\cos \angle(V, \widetilde{V})=\left\|V^{T} \widetilde{V}\right\|_{2}$ [36]. The following theorem shows why we can solve the matrix exponential eigenproblems approximately in the EDA framework.

Theorem 6. Let $V, \widetilde{V} \in \mathbb{R}^{d \times t}$ be orthonormal matrices whose columns are the "exact" and "computed" solutions of (3.1) or (3.12), respectively. Denote by $d_{i j}=\left\|V^{T}\left(\widehat{\mathbf{x}}_{i}-\widehat{\mathbf{y}}_{j}\right)\right\|_{2}$ and $\widetilde{d}_{i j}=\left\|\widetilde{V}^{T}\left(\widehat{\mathbf{x}}_{i}-\widehat{\mathbf{y}}_{j}\right)\right\|_{2}$ the "exact" and the 
“computed" Euclidean distances, respectively, and let $\sin \angle(V, \widetilde{V})=\left\|\left(I-V V^{T}\right) \widetilde{V}\right\|_{2}$ be the distance between the eigenspace $\operatorname{span}\{V\}$ and the approximate eigenspace $\operatorname{span}\{\widetilde{V}\}$. If $\left\|\widehat{\mathbf{x}}_{i}\right\|_{2},\left\|\widehat{\mathbf{y}}_{j}\right\|_{2}=1$ and $\cos \angle(V, \widetilde{V}) \neq 0$, then

$$
\frac{\widetilde{d}_{i j}-2 \sin \angle(V, \widetilde{V})}{\cos \angle(V, \widetilde{V})} \leq d_{i j} \leq \widetilde{d}_{i j} \cos \angle(V, \widetilde{V})+2 \sin \angle(V, \widetilde{V}) .
$$

Proof. See the Appendix.

Remark 3. Theorem 6 indicates that if $\sin \angle(V, \widetilde{V})$ is sufficiently small (say, in the order of $\left.10^{-4}\right)$, then the $\left\{d_{i j}\right\}^{\prime} s$ and the $\left\{\widetilde{d}_{i j}\right\}^{\prime} s$ will be close to each other. As a result, there is no need to compute the eigenvectors too accurately. This explains why the matrix exponential eigenproblems (3.1) and (3.12) can be solved approximately in practice. We stress that our result also applies to the case of $\left\|\widehat{\mathbf{x}}_{i}\right\|_{2},\left\|\widehat{\mathbf{y}}_{j}\right\|_{2} \leq 1$, and it is very important to have normalized data when dealing with exponentials; see the numerical experiments made in Section IV. Further, instead of using 2-norm, one can establish other bounds in terms of some commonly used norms.

However, we cannot get the value of $\sin \angle(V, \widetilde{V})$ a priori, since the "exact" eigenspace $\operatorname{span}\{V\}$ is unavailable. Let $\left(\lambda_{1}, \mathbf{x}_{1}\right), \ldots,\left(\lambda_{t}, \mathbf{x}_{t}\right)$ be the desired eigenpairs, it was shown that if the minimal distance between the Ritz values $\widetilde{\lambda}_{1}, \widetilde{\lambda}_{2}, \ldots, \widetilde{\lambda}_{t}$ and the other eigenvalues (i.e., the eigenvalues other than $\widetilde{\lambda}_{1}, \widetilde{\lambda}_{2}, \ldots, \widetilde{\lambda}_{t}$ ) is sufficiently large, then $\sin \angle(V, \widetilde{V})$ is proportional to the residual norms of Ritz pairs [18], [36]. Therefore, we can use the largest residual norm (see Algorithms 2,3 for the definition of residual norm) of the Ritz pairs to take the place of $\sin \angle(V, \widetilde{V})$ in practice. Experimentally, we find that a tolerance of $\varepsilon=10^{-4}$ is good enough.

In summary, we propose the main algorithm of this work for solving the large scale matrix exponential eigenproblems.

\section{Algorithm 5. Krylov-EDA algorithms with inexact implementation for matrix exponential discriminant analysis}

Steps 1-4 are the same as those of Algorithm 4.

5. Given a convergence threshold $\varepsilon\left(\right.$ e.g., $\left.\varepsilon=10^{-4}\right)$, compute the desired eigenvectors by using a restarted Krylov subspace algorithm (e.g. [35]) for solving (3.1) (by using the Arnoldi algorithm) or (3.13) (by using the Lanczos algorithm), where we use steps $6 A-10 \mathrm{~A}$ or $6 \mathrm{~L}-12 \mathrm{~L}$ in Algorithm 4 for matrix exponential-vector products.

6. Orthogonalize the columns of the projection matrix $V$.

Remark 4. In practical implementations, the "Preprocessing" phase 1-4 are run once for all, and the variables $Q_{B}, Q_{W}, \Sigma_{B}$ and $\Sigma_{W}$ are stored for a latter use. In this procedure, one needs to perform two skinny $Q R$ decompositions in $\mathcal{O}\left(d K^{2}\right)$ and $\mathcal{O}\left(d n^{2}\right)$ flops [14], respectively. Further, it is required to perform SVD of two small-sized matrices $R_{B}$ and $R_{W}$, in $\mathcal{O}\left(K^{3}\right)$ and $\mathcal{O}\left(n^{3}\right)$ flops [14], respectively; as well as to compute a smallsized diagonal matrix exponential in $\mathcal{O}(K)$ flops [15], which are negligible as $K \leq n \ll d$. In Step 5, the main overhead is the computation of matrix exponential-vector products (see 6A-10A or 6L-12L in Algorithm 4), as well as the orthgonlaization procedure in Arnoldi or Lanczos process. Thus, as $K \leq n \ll d$, the main cost of Algorithm 5 is $\mathcal{O}(d)$.

On the other hand, if the $n$ training vectors are linear independent, the ranks of the matrices $S_{B}$ and $S_{W}$ are $K-1$ and $n-K$, respectively, and the main storage requirement of Algorithm 5 is to store $n-1$ vectors of length $d$. Therefore, the computational complexity of our new algorithm is much less than those of Algorithm 1.

Although our new algorithms belong to the class of inexact Krylov subspace algorithms, they are essentially different from the inexact inner-outer Krylov subspace methods with inexact matrix-vector products [33]. In those methods, the tolerance of the inner iterative process (i.e., approximating the matrix exponential action) can be significantly relaxed once the outer process (i.e, the Arnoldi/Lanczos eigenvalue solver) has started to converge. However, in our new algorithms the matrix exponential-vector products are computed "exactly" based on the closed- 
form formulae. Further, the tolerance for Arnoldi/Lanczos eigenvalue solver in the new algorithm is determined in advance.

\section{NUMERICAL EXPERIMENTS}

In this section, we perform some numerical experiments to illustrate the efficiency of Algorithm 5 for face recognition. All the numerical experiments were run on a Dell PC with eight core Intel(R) Core(TM)i7-2600 processor with CPU $3.40 \mathrm{GHz}$ and RAM 16.0 GB, under the Windows 7 with 64-bit operating system. All the numerical results were obtained from using a MATLAB 7.10.0 implementation. In all the experiments, a random subset with $\ell$ images per subject is taken to form the training set, and the rest of the images are used as the testing set, and 10 random splits are run so that one can obtain a stable recognition result. Each column $\mathbf{a}_{i}$ of the data matrices is scaled by its 2-norm.

We apply the nearest neighbor (NN) [8] as the classifier with the $L_{2}$ metric as distance measure. In all the algorithms for comparison, the projection matrix $V$ is composed of the first $K-1$ (i.e., $t=K-1$ ) dominant discriminant vectors, where $K$ is the number of classes. In all the EDA-based algorithms, the columns of $V$ are orthonomalized by using the MATLAB built-in function orth.m, where the orth operation is performed via a QR decomposition, i.e., stabilized Gram-Schmidt.

For the performance of the EDA-based methods and how they compare with the current state-of-the-art, we refer to [9], [39], [40], [45], [50]. Indeed, if the large matrix exponential eigenproblems (1.6) and (3.13) were solved "exactly", Algorithm 5 is mathematically equivalent to the original EDA method proposed in [50]. The main aim of this section is to show that Algorithm 5 runs much faster than Algorithm 1, while the classification accuracy of the former is comparable to that of the latter.

The different EDA-based algorithms are described as follows:

- Arnoldi-EDA (Algorithm 5): We apply Algorithm 5 to solve the large nonsymmetric matrix exponential eigenproblem (3.1), in which the implicitly restarted Arnoldi algorithm [35] (the MATLAB built-in function eigs.m) is used. The matrix exponential-vector products are computed by using Algorithm 4. The stopping criterion for the large eigenproblem is chosen as $\varepsilon=10^{-4}$.

- Lanczos-EDA (Algorithm 5): We apply Algorithm 5 for solving the large symmetric matrix exponential eigenproblem (3.13), in which the implicitly restarted Lanczos algorithm [35] (the MATLAB built-in function eigs.m) is used. The matrix exponential-vector products are computed by using Algorithm 4. The stopping criterion for the large eigenproblem is chosen as $\varepsilon=10^{-4}$.

- EDA-eigs: Solving the generalized symmetric matrix exponential eigenproblem (1.6) by using the MATLAB built-in function eigs.m, the convergence threshold is chosen as $\varepsilon=10^{-8}$. This algorithm mimics forming the two matrix exponentials $\exp \left(S_{B}\right)$ and $\exp \left(S_{W}\right)$ explicitly, while solving the matrix exponential eigenproblem iteratively with a relatively higher accuracy. The matrix exponentials are evaluated by using the MATLAB built-in function expm.m.

- EDA (Algorithm 1): The EDA method proposed in [50], in which we form $\exp \left(-S_{W}\right) \exp \left(S_{B}\right)$ and solve the nonsymmetric matrix exponential eigenproblem (3.1) by using the implicit QR algorithm [3], [14] (the MATLAB built-in function eig.m). The matrix exponentials are computed by using the MATLAB built-in function expm.m.

Example 4.1 In this example, we compare the EDA-based algorithms with LDA+PCA [4] and the classical LDA method [10], [12], and show the efficiency of the exponential discriminant analysis method. A subset of AR database [20] is used here with 1680 face images from 120 persons (14 images per person), and all images are cropped and scaled to $50 \times 40$. Figure 4.1 presents the sample of cropped AR database images of three individuals. A random subset with $\ell=2,3,5$ images per subject is taken to form the training set, and the rest of the images are used as the testing set. We run Arnoldi-EDA, Lanczos-EDA, EDA-eigs, EDA and LDA+PCA on this problem. As a by-product, we also list the numerical results of the "Classical LDA" method [10], [12], i.e., the classical LDA method with the QZ algorithm [14] for the generalized eigenproblem (1.4) (by using the MATLAB built-in function eig.m). As was done in [50], for LDA+PCA, we preserve $99 \%$ energy in the PCA stage, followed by 




Figure 4.1, Example 4.1: Sample face images of 3 individuals from the AR database, $d=50 \times 40$.

LDA. Table 4.1 lists the CPU time in seconds, recognition accuracy and standard derivations obtained from the different algorithms. When $\ell=3$, we plot in Figure 4.2 recognition accuracy of the six algorithms with respect to the projected dimension on the AR database. Notice that the curves of Arnoldi-EDA, EDA-eigs, and EDA overlap with each other.

\begin{tabular}{lccc}
\hline Algorithm & 2 Train & 3 Train & 5 Train \\
\hline Arnoldi-EDA & $0.52(84.3 \% \pm 1.3 \%)$ & $0.71(92.0 \% \pm 1.0 \%)$ & $1.21(97.0 \% \pm 1.4 \%)$ \\
Lanczos-EDA & $0.57(84.6 \% \pm 1.9 \%)$ & $0.82(92.2 \% \pm 1.0 \%)$ & $1.51(97.3 \% \pm 1.2 \%)$ \\
EDA-eigs & $5.74(84.3 \% \pm 1.3 \%)$ & $6.06(92.0 \% \pm 1.0 \%)$ & $6.71(97.0 \% \pm 1.4 \%)$ \\
EDA & $9.10(84.3 \% \pm 1.3 \%)$ & $8.85(92.0 \% \pm 1.0 \%)$ & $9.14(97.0 \% \pm 1.4 \%)$ \\
\hline LDA+PCA & $0.09(82.6 \% \pm 1.9 \%)$ & $0.20(87.7 \% \pm 1.8 \%)$ & $0.43(94.8 \% \pm 1.2 \%)$ \\
Classical LDA & $53.2(65.0 \% \pm 22.2 \%)$ & $53.1(71.3 \% \pm 11.5 \%)$ & $52.7(78.0 \% \pm 5.9 \%)$ \\
\hline
\end{tabular}

Table 4.1, Example 4.1: CPU time in seconds, recognition accuracy and standard derivation (in brackets) of the six algorithms on the AR database, $d=50 \times 40, K=120$. Here " $X$ train" means a random subset with $\ell=X$ images per subject is taken to form the training set, and the rest of the images are used as the testing set.

We see from Table 4.1 that the recognition accuracy obtained from the four EDA-based algorithms are about the same, which are (much) higher than those from LDA+PCA and Classical LDA. Furthermore, both Arnoldi-EDA and Lanczos-EDA run much faster than EDA-eigs and EDA, while EDA-eigs performs better than EDA. The classical LDA method works the poorest in terms of CPU time and recognition accuracy. This is due to the fact that the dimension $d=2000>n=1680$, the number of samples in this example. So we suffer from the SSS problem. Recall that the classical LDA method fail to cure this drawback properly.

Example 4.2 In this example, we show that our new algorithms Arnoldi-EDA and Lanczos-EDA run much faster than EDA and EDA-eigs, while keeping comparable recognition accuracy. There are two data sets in this example. The first one is the Yale face database taken from the Yale Center for Computational Vision and Control. It contains 165 grayscale images of $K=15$ individuals. The images demonstrate variation with the following expressions or configurations: 1) lighting: center light, left light, and right light; 2) with/without glasses; and 3) facial expressions: normal, happy, sad, sleepy, surprised, and winkling. The original image size is $320 \times 243$ pixels. Figure 4.3 gives the sample of cropped Yale database images of three individuals.

The second test set is the Extended YaleB database. This database contains 5760 single light source images of 10 subjects each seen under 576 viewing conditions ( 9 different poses and 64 illumination conditions of each person). The images have normal, sleepy, sad and surprising expressions. There are some images with or without glasses. These images are captured by varying the position of light source at the center, left or right. For every subject in a particular pose, an image with ambient (background) illumination was also captured. A subset of 


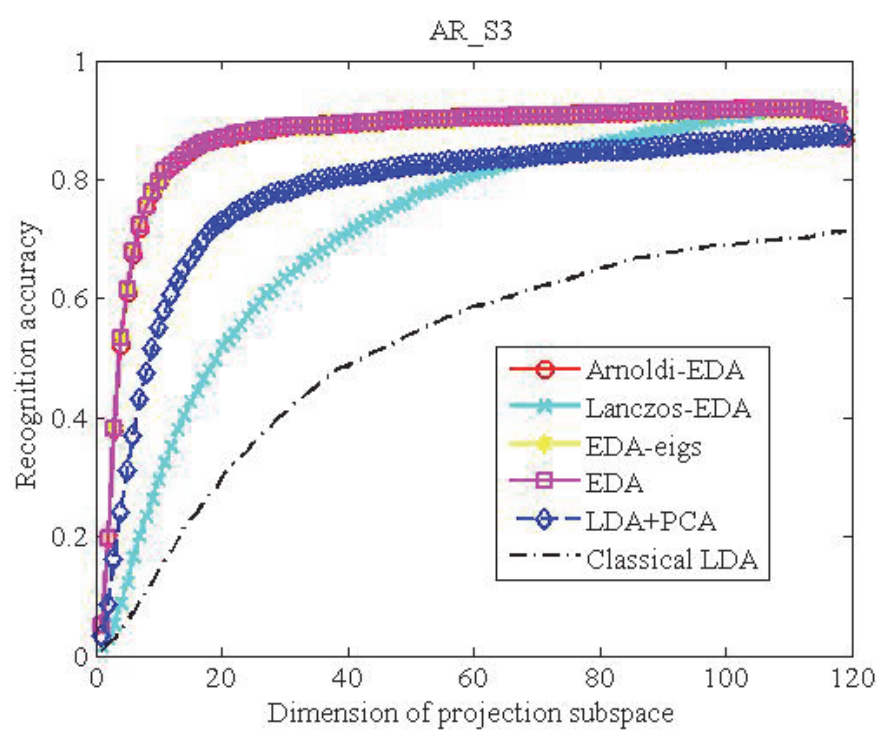

Figure 4.2, Example 4.1: Recognition accuracy with respect to the projected dimension on the AR database, 3 Train. Notice that the curves of Arnoldi-EDA, EDA-eigs, and EDA overlap with each other.



Figure 4.3, Example 4.2: Sample face images of 3 individuals from the Yale database, $d=64 \times 64$.

$k=38$ with 2432 images are used in this example (64 images of per individual with illumination). Figure 4.4 shows the sample of cropped Extended YaleB database images of three individuals.

In this example, all images are aligned based on eye coordinates and are cropped and scaled to $32 \times 32,64 \times 64$ and $100 \times 100$, respectively. A random subset with $\ell=3,5,8$ images per subject is taken to form the training set, and the rest of the images are used as the testing set. Tables 4.2 and 4.3 report the CPU time in seconds, recognition accuracy and standard derivation of different algorithms.

Three remarks are given. First, our new algorithms Arnoldi-EDA and Lanczos-EDA outperform EDA-eigs and EDA considerably in terms of CPU time, while the recognition accuracy and standard derivation of the four algorithms are comparable. For example, we see from Table 4.2 that when $d=100 \times 100$ and $\ell=3$, ArnoldiEDA and Lanczos-EDA used 0.10 and 0.09 seconds, while EDA-eigs and EDA used 377.8 and 996.8 seconds, respectively. Thus, both the Arnoldi-EDA and the Lanczos-EDA algorithms circumvent the drawback of large overhead that exists in the original EDA algorithm, while keeping comparable recognition accuracy. Second, cropping the original images may lose some useful information and thus may result in a low recognition accuracy. For instance, for the Yale database, when $\ell=5$, the recognition accuracy of Arnoldi-EDA applying to $d=100 \times 100$ images is $93.9 \%$, while the recognition accuracy is only $72.9 \%$ as $d=32 \times 32$. Third, it is seen from Tables 

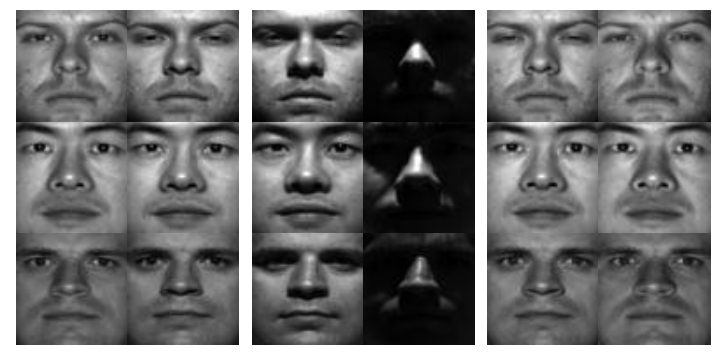

Figure 4.4, Example 4.2: Sample face images of 3 individuals from the Extended YaleB database, $d=64 \times 64$.

4.2-4.3 that we cannot tell which one, Arnoldi-EDA or Lanczos-EDA, is definitely better than the other in terms of both CPU time and recognition accuracy. We believe the best one is dataset dependent, which makes it difficult to choose the best suited algorithm.

\begin{tabular}{lcccc}
\hline Algorithm & $d$ & 3 Train & 5 Train & 8 Train \\
\hline Arnoldi-EDA & $32 \times 32$ & $0.02(64.0 \% \pm 4.4 \%)$ & $0.03(72.9 \% \pm 3.7 \%)$ & $0.04(82.4 \% \pm 5.6 \%)$ \\
Lanczos-EDA & $32 \times 32$ & $0.02(64.1 \% \pm 2.6 \%)$ & $0.02(72.4 \% \pm 3.9 \%)$ & $0.04(81.6 \% \pm 5.6 \%)$ \\
EDA-eigs & $32 \times 32$ & $0.68(64.2 \% \pm 4.5 \%)$ & $0.70(73.0 \% \pm 3.3 \%)$ & $0.76(82.9 \% \pm 5.7 \%)$ \\
EDA & $32 \times 32$ & $1.14(64.2 \% \pm 4.5 \%)$ & $1.16(73.0 \% \pm 3.3 \%)$ & $1.14(82.9 \% \pm 5.7)$ \\
\hline Arnoldi-EDA & $64 \times 64$ & $0.05(74.8 \% \pm 4.0 \%)$ & $0.06(83.4 \% \pm 3.1 \%)$ & $0.11(90.2 \% \pm 1.9 \%)$ \\
Lanczos-EDA & $64 \times 64$ & $0.04(75.1 \% \pm 4.3 \%)$ & $0.06(84.0 \% \pm 5.3 \%)$ & $0.11(90.9 \% \pm 2.5 \%)$ \\
EDA-eigs & $64 \times 64$ & $32.1(75.3 \% \pm 4.5 \%)$ & $32.1(83.8 \% \pm 4.1 \%)$ & $28.8(90.7 \% \pm 3.9 \%)$ \\
EDA & $64 \times 64$ & $76.4(75.3 \% \pm 4.5 \%)$ & $76.5(83.8 \% \pm 4.1 \%)$ & $72.6(90.7 \% \pm 3.9 \%)$ \\
Arnoldi-EDA & $100 \times 100$ & $0.10(89.3 \% \pm 2.4 \%)$ & $0.15(93.9 \% \pm 3.4 \%)$ & $0.26(96.4 \% \pm 3.3 \%)$ \\
Lanczos-EDA & $100 \times 100$ & $0.09(89.6 \% \pm 2.7 \%)$ & $0.16(93.7 \% \pm 4.2 \%)$ & $0.28(96.0 \% \pm 3.3 \%)$ \\
EDA-eigs & $100 \times 100$ & $377.8(89.3 \% \pm 2.4 \%)$ & $370.7(94.1 \% \pm 3.4 \%)$ & $415.0(96.9 \% \pm 3.3 \%)$ \\
EDA & $100 \times 100$ & $996.8(89.3 \% \pm 2.4 \%)$ & $984.1(94.1 \% \pm 3.4 \%)$ & $1031.5(96.9 \% \pm 3.3 \%)$ \\
\hline
\end{tabular}

Table 4.2, Example 4.2: CPU time in seconds, recognition accuracy and standard derivation (in brackets) of the four algorithms on the Yale database, $K=15$. Here " $X$ train" means a random subset with $\ell=X$ images per subject is taken to form the training set, and the rest of the images are used as the testing set.

\begin{tabular}{lcccc}
\hline Algorithm & $d$ & 3 Train & 5 Train & 8 Train \\
\hline Arnoldi-EDA & $32 \times 32$ & $0.06(55.6 \% \pm 3.6 \%)$ & $0.09(73.0 \% \pm 1.3 \%)$ & $0.12(82.5 \% \pm 1.7 \%)$ \\
Lanczos-EDA & $32 \times 32$ & $0.06(59.3 \% \pm 2.3 \%)$ & $0.09(71.6 \% \pm 1.5 \%)$ & $0.15(82.5 \% \pm 1.8 \%)$ \\
EDA-eigs & $32 \times 32$ & $0.94(56.4 \% \pm 4.1 \%)$ & $1.10(73.6 \% \pm 1.5 \%)$ & $1.21(83.4 \% \pm 1.9 \%)$ \\
EDA & $32 \times 32$ & $1.18(56.4 \% \pm 4.1 \%)$ & $1.22(73.6 \% \pm 1.5 \%)$ & $1.28(83.4 \% \pm 1.9 \%)$ \\
\hline Arnoldi-EDA & $64 \times 64$ & $0.20(56.5 \% \pm 3.6 \%)$ & $0.41(73.0 \% \pm 1.7 \%)$ & $0.75(82.5 \% \pm 1.6 \%)$ \\
Lanczos-EDA & $64 \times 64$ & $0.23(59.1 \% \pm 1.8 \%)$ & $0.41(71.5 \% \pm 1.8 \%)$ & $0.92(82.5 \% \pm 1.7 \%)$ \\
EDA-eigs & $64 \times 64$ & $32.7(57.0 \% \pm 3.7 \%)$ & $33.9(73.6 \% \pm 1.8 \%)$ & $39.3(83.7 \% \pm 1.6 \%)$ \\
EDA & $64 \times 64$ & $73.9(57.0 \% \pm 3.7 \%)$ & $74.1(73.6 \% \pm 1.8 \%)$ & $78.4(83.7 \% \pm 1.6 \%)$ \\
Arnoldi-EDA & $100 \times 100$ & $0.56(56.2 \% \pm 5.7 \%)$ & $1.33(73.6 \% \pm 1.4 \%)$ & $1.68(83.1 \% \pm 1.7 \%)$ \\
Lanczos-EDA & $100 \times 100$ & $0.77(59.5 \% \pm 2.8 \%)$ & $1.79(71.5 \% \pm 1.8 \%)$ & $2.14(83.2 \% \pm 1.7 \%)$ \\
EDA-eigs & $100 \times 100$ & $399.4(57.3 \% \pm 5.2 \%)$ & $400.3(73.7 \% \pm 1.8 \%)$ & $452.3(84.2 \% \pm 1.8 \%)$ \\
EDA & $100 \times 100$ & $984.7(57.3 \% \pm 5.2 \%)$ & $983.8(73.7 \% \pm 1.8 \%)$ & $1028.9(84.2 \% \pm 1.8 \%)$ \\
\hline
\end{tabular}

Table 4.3, Example 4.2: CPU time in seconds, recognition accuracy and standard derivation (in brackets) of the four algorithms on the Extended YaleB database, $K=38$. Here " $X$ train" means a random subset with $\ell=X$ images per subject is taken to form the training set, and the rest of the images are used as the testing set. 


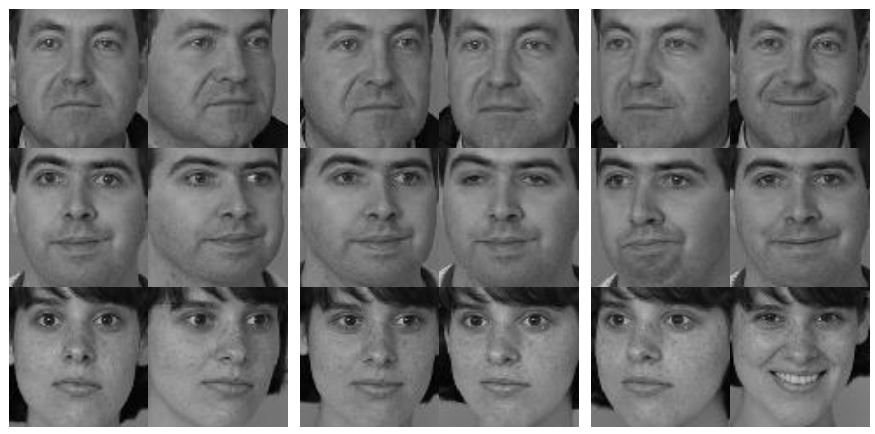

Figure 4.5, Example 4.3 : Sample face images of 3 individuals from the FERET database, $d=80 \times 80$.

Example 4.3 In this example, we show the efficiency of our "inexact" strategy for solving the large matrix exponential eigenproblems (3.1) and (3.13). The FERET database is one of the standard image database specially used for the face recognition algorithms [29]. The final corpus consists of 14051 eight-bit grayscale images of human heads with views ranging from frontal to left and right profiles. In this example, we consider a subset of 1400 images of $K=200$ individuals, in which each person contributing seven images. The seven images of each individual consist of different illumination and expression variation. Before experiment, the facial images are cropped to size of $80 \times 80$ pixels. Figure 4.5 lists the sample of cropped FERET database images of three individuals. A random subset with $\ell=2,3,5$ images per subject is taken to form the training set, and the rest of the images are used as the testing set. In this example, we have to compute $K-1=199$ dominant eigenpairs of a $6400 \times 6400$ matrix using Arnoldi-EDA and Lanczos-EDA, which is a challenging task.

\begin{tabular}{lcccc}
\hline Algorithm & $\varepsilon$ & 2 Train & 3 Train & 5 Train \\
\hline Arnoldi-EDA & $10^{-2}$ & $5.16(62.4 \% \pm 1.7 \%)$ & $6.53(73.3 \% \pm 1.6 \%)$ & $10.1(81.1 \% \pm 1.4 \%)$ \\
& $10^{-4}$ & $5.22(62.4 \% \pm 1.7 \%)$ & $6.64(73.3 \% \pm 1.6 \%)$ & $10.1(81.2 \% \pm 1.4 \%)$ \\
& $10^{-6}$ & $5.16(62.4 \% \pm 1.7 \%)$ & $10.5(73.4 \% \pm 1.6 \%)$ & $18.8(81.2 \% \pm 1.4 \%)$ \\
& $10^{-8}$ & $5.18(62.4 \% \pm 1.7 \%)$ & $11.1(73.4 \% \pm 1.6 \%)$ & $24.1(81.4 \% \pm 1.4 \%)$ \\
& $10^{-10}$ & $5.17(62.3 \% \pm 1.7 \%)$ & $11.5(73.4 \% \pm 1.6 \%)$ & $26.7(81.3 \% \pm 1.4 \%)$ \\
\hline Lanczos-EDA & $10^{-2}$ & $6.31(44.2 \% \pm 2.3 \%)$ & $8.48(61.6 \% \pm 1.5 \%)$ & $13.6(79.1 \% \pm 1.9 \%)$ \\
& $10^{-4}$ & $6.44(44.2 \% \pm 2.3 \%)$ & $8.52(61.7 \% \pm 1.5 \%)$ & $13.7(78.9 \% \pm 1.9 \%)$ \\
& $10^{-6}$ & $6.38(44.2 \% \pm 2.3 \%)$ & $14.0(61.6 \% \pm 1.5 \%)$ & $26.5(79.2 \% \pm 1.9 \%)$ \\
& $10^{-8}$ & $6.34(44.2 \% \pm 2.3 \%)$ & $15.4(61.6 \% \pm 1.5 \%)$ & $34.2(79.2 \% \pm 1.9 \%)$ \\
& $10^{-10}$ & $6.34(44.2 \% \pm 2.3 \%)$ & $16.1(61.6 \% \pm 1.5 \%)$ & $38.9(79.2 \% \pm 1.9 \%)$ \\
\hline EDA & - & $271.0(62.4 \% \pm 1.7 \%)$ & $265.5(73.5 \% \pm 1.7 \%)$ & $268.1(81.4 \% \pm 1.7 \%)$ \\
LDA+PCA & - & $0.53(42.3 \% \pm 2.3 \%)$ & $1.12(39.7 \% \pm 1.7 \%)$ & $2.43(57.5 \% \pm 2.0 \%)$ \\
\hline
\end{tabular}

Table 4.4, Example 4.3 : CPU time in seconds, recognition accuracy and standard derivation (in brackets) of the Arnoldi-EDA and Lanczos-EDA algorithms (with different tolerances for exponential eigenproblems) on the FERET database, $d=80 \times 80, K=200$. Here " $X$ train" means a random subset with $\ell=X$ images per subject is taken to form the training set, and the rest of the images are used as the testing set.

In order to show the efficiency of our inexact strategy, we run the (implicitly restarted) Arnoldi and Lanczos algorithm in Step 5 of Algorithm 5 with convergence tolerance $\varepsilon=10^{-2}, 10^{-4}, 10^{-6}, 10^{-8}$ and $10^{-10}$, respectively. Table 4.4 presents the numerical results. We observe that the two inexact algorithms work quite well in all the cases, and a tolerance of $\varepsilon=10^{-4}$ or $10^{-2}$ is good enough. When $\ell=2$, the matrix exponential eigenproblems are (relatively) easy to solve, and the CPU time used for different tolerances are about the same. As $\ell=3$ and 5, the matrix exponential eigenproblems become hard to solve, and Arnoldi-EDA or Lanczos-EDA with $\varepsilon=10^{-4}$ or 



Figure 4.6, Example 4.4: Sample face images of 3 individuals from the CMU-PIE database, $d=640 \times 486$.

$10^{-2}$ can save about half of the CPU time than they with $\varepsilon=10^{-10}$. It is observed that Algorithm 5 outperforms Algorithm 1 significantly according to CPU time, while the classification accuracy of the former is comparable to that of the latter. That is, as is indicated by Theorem 6 , it is unnecessary to solve the large exponential eigenproblems in a very high accuracy; see Remark 3. This is favorable to very large matrix computations arising from high dimensionality reduction problems. As a comparison, we also list the numerical results obtained from running EDA and LDA+PCA. One observes that Arnoldi-EDA runs much faster than EDA, and it is superior to LDA+PCA in terms of recognition accuracy.

For this problem, however, we notice that the recognition accuracies of Lanczos-EDA are much lower than those of Arnoldi-EDA and EDA. We don't know why this happens and it deserves further investigation. One possible reason is that the approximation $y$ obtained from Lanczos-EDA undergoes a exponential transformation to get the desired solution $\mathbf{x}$, see (3.14). Another possible reason is that the results of recognition are closely related to the classifier utilized.

Example 4.4 In this example, we demonstrate that Arnoldi-EDA and Lanczos-EDA can be applied to data sets with very high dimension. The CMU-PIE database contains more than 40,000 images of 68 subjects with more than 500 images for each. These face images are captured by 13 synchronized cameras and 21 flashes under varying pose, illumination, expression and lights. In this experiment, we choose $K=10$ subjects and 10 images under different illuminations, lights, expressions and poses for each subject. Thus, the total number of images chosen from CMU-PIE database is $n=100$. The image size is $d=486 \times 640$ pixels, so we have to deal with eigenproblems of size $311,040 \times 311,040$. We randomly select with $\ell=3,5,8$ images for each subject to organize a training set, while the testing set consists of the remaining images. Figure 4.6 depicts the sample of CMU-PIE database images of three individuals.

In this example, we compare the inexact Krylov subspace algorithms Arnoldi-EDA and Lanczos-EDA with some state-of-the-art algorithms including EDA[50], PCA [37], LDA+PCA (Fisherfaces) [4], NLDA (the null space LDA method) [5], [6], [19], LDA/QR [48], LDA/GSVD [16], [47], RLDA [13] (the regularized LDA method, where the regularized parameter is chosen as 0.01) for the face recognition problem. We preserve $99 \%$ energy for recognition for PCA. For LDA+PCA, we also preserve $99 \%$ energy in the PCA stage, followed by LDA. For the NLDA method, we use the implementation advocated in [19]. However, this NLDA implementation only applies to data matrix whose columns are linearly independent [19]. Table 4.5 lists the numerical results.

It is seen from Table 4.5 that for very large data sets such as CMU-PIE, all the algorithms EDA-eigs, EDA, RLDA, and LDA/GSVD fail to work, due to their heavy storage requirements and computational cost. Indeed, in EDA, EDA-eigs, RLDA and LDA/GSVD, one has to explicitly form and store some matrices of size $d \times d$. A more powerful computer is needed for running these algorithms. As a comparison, Arnoldi-EDA and LanczosEDA perform quite well in all the situations, and their recognition accuracy is a little higher than those of PCA. Consequently, our new algorithms are suitable to data sets with high dimension, and they are competitive alternatives 
to EDA for high dimensionality reduction problems.

On the other hand, we see that PCA, LDA+PCA, LDA/QR and NLDA run faster than Arnoldi-EDA and LanczosEDA for this example. The reason is possibly that the number of samples $n$ (which is only 100) and the number of classes $K$ are relatively small in this example. In other words, Arnoldi-EDA and Lanczos-EDA seem to work well for some databases and not for others, and the recognition accuracy obtained from the EDA-type algorithms may be lower than those from the other algorithms. So it is difficult to choose the most suited algorithm in general, and the best choice is often problem-dependent.

\begin{tabular}{lccc}
\hline Algorithm & 3 Train & 5 Train & 8 Train \\
\hline Arnoldi-EDA & $2.11(88.7 \% \pm 4.6 \%)$ & $3.42(91.8 \% \pm 3.6 \%)$ & $6.00(92.5 \% \pm 5.9 \%)$ \\
Lanczos-EDA & $2.57(90.6 \% \pm 3.6 \%)$ & $4.24(91.4 \% \pm 2.3 \%)$ & $7.22(92.5 \% \pm 5.9 \%)$ \\
EDA-eigs & - & - & - \\
EDA & - & - & - \\
\hline LDA+PCA & $0.41(90.3 \% \pm 4.1 \%)$ & $0.78(92.6 \% \pm 3.3 \%)$ & $1.23(93.0 \% \pm 5.9 \%)$ \\
PCA & $0.33(83.4 \% \pm 6.4 \%)$ & $0.71(87.8 \% \pm 3.6 \%)$ & $1.11(91.0 \% \pm 7.4 \%)$ \\
RLDA & - & - & - \\
NLDA & $1.12(91.1 \% \pm 2.9 \%)$ & $2.74(93.2 \% \pm 3.2 \%)$ & $6.49(93.5 \% \pm 6.3 \%)$ \\
LDA/QR & $0.49(89.0 \% \pm 4.9 \%)$ & $0.65(91.6 \% \pm 4.4 \%)$ & $0.92(92.5 \% \pm 5.9 \%)$ \\
LDA/GSVD & - & - & - \\
\hline
\end{tabular}

Table 4.5, Example 4.4: CPU time in seconds, recognition accuracy and standard derivation (in brackets) of the 10 algorithms on the CMU-PIE database, $d=640 \times 486, K=10$. Here " $X$ train" means a random subset with $\ell=X$ images per subject is taken to form the training set, and the rest of the images are used as the testing set. Here "-" means the algorithm suffers from the difficulty of "Out of Memory".

\section{CONCLUSIONS AND FUTURE WORK}

The computation of large scale matrix exponential eigenproblem is the bottleneck in the framework of EDA method for high dimensionality reduction problems. In this paper, we propose two new algorithms to speed up the EDA method significantly while keeping comparable recognition accuracy, so that the EDA framework can be applied to data sets with very high dimensionality. The new algorithms are based on Krylov subspace projection techniques and inexact solvers, in which the matrix exponentials need not to be formed or stored explicitly, and the eigenpairs are computed only approximately. Some new results are given to compare the discriminant analysis criterion of EDA and that of LDA. It is shown that the EDA-type methods have more discriminant power than the original LDA method. This coincides with the numerical results given in [1], [9], [39], [40], [45], [50]. Furthermore, the proposed Arnoldi-EDA and Lanczos-EDA algorithms outperform the EDA method considerably in terms of CPU time, while the classification accuracy and standard derivation of the new algorithms are comparable to those of the latter.

We point out that our new strategies also apply to other exponential based methods such as the exponential Laplacian embedding, exponential LDE, exponential LPP, exponential discriminant regularization, and 2DEDA for high dimensionality reduction other than face recognition. How to combine our new strategies with other efficient eigen-solvers deserves further investigation. For instance, the Jacobi-Davidson method [34] is a successful algorithm for large eigenproblems. This method is applicable to the matrix exponential eigenproblems. Furthermore, our inexact strategy only relates the perturbation of the projections to the perturbation of the projected points for the nearest neighbor $(\mathrm{NN})$. It is meaningful to establish a performance bound for general KNN which should also involve the distances between points of different classes. They are interesting topics and are definitely a part of our future work. 


\section{ACKNOWLEDGMENTS}

We would like to express our sincere thanks to the three referees for their insightful comments and invaluable suggestions that greatly improved the representation of this paper.

\section{APPENDIX}

\section{A. Proof of Theorem 1}

We only prove (3.7), and the proof of (3.8) is similar. It follows from (1.1), (1.2) and (3.3)-(3.6) that

$$
\begin{aligned}
S_{B} & =H_{B} H_{B}^{T}=\left(Q_{B} U_{B}\right) \Sigma_{B}\left(V_{B}^{T} V_{B}\right) \Sigma_{B}\left(U_{B}^{T} Q_{B}^{T}\right)=\widetilde{Q}_{B} D_{B} \widetilde{Q}_{B}^{T} \\
& =\left[\begin{array}{ll}
\widetilde{Q}_{B} & \widetilde{Q}_{B}^{\perp}
\end{array}\right]\left[\begin{array}{cc}
D_{B} & \\
& \mathbf{0}_{(d-k) \times(d-k)}
\end{array}\right]\left[\begin{array}{c}
\widetilde{Q}_{B}^{T} \\
\left(\widetilde{Q}_{B}^{\perp}\right)^{T}
\end{array}\right] .
\end{aligned}
$$

From the properties of matrix function [15, Theorem 1.13], we obtain

$$
\begin{aligned}
f\left(S_{B}\right) & =\left[\begin{array}{ll}
\widetilde{Q}_{B} & \widetilde{Q}_{B}^{\perp}
\end{array}\right]\left[\begin{array}{ll}
f\left(D_{B}\right) & \\
& f\left(\mathbf{0}_{(d-k) \times(d-k)}\right)
\end{array}\right]\left[\begin{array}{c}
\widetilde{Q}_{B}^{T} \\
\left(\widetilde{Q}_{B}^{\perp}\right)^{T}
\end{array}\right] \\
& =\left[\begin{array}{ll}
\widetilde{Q}_{B} f\left(D_{B}\right) & \widetilde{Q}_{B}^{\perp} f\left(\mathbf{0}_{(d-k) \times(d-k)}\right)
\end{array}\right]\left[\begin{array}{c}
\widetilde{Q}_{B}^{T} \\
\left(\widetilde{Q}_{B}^{\perp}\right)^{T}
\end{array}\right] \\
& =\widetilde{Q}_{B} f\left(D_{B}\right) \widetilde{Q}_{B}^{T}+\widetilde{Q}_{B}^{\perp} f\left(\mathbf{0}_{(d-k) \times(d-k)}\right)\left(\widetilde{Q}_{B}^{\perp}\right)^{T} .
\end{aligned}
$$

And (3.9)-(3.11) follow from

$$
\widetilde{Q}_{B}^{\perp}\left(\widetilde{Q}_{B}^{\perp}\right)^{T}=I-\widetilde{Q}_{B} \widetilde{Q}_{B}^{T}, \widetilde{Q}_{W}^{\perp}\left(\widetilde{Q}_{W}^{\perp}\right)^{T}=I-\widetilde{Q}_{W} \widetilde{Q}_{W}^{T},
$$

and the fact that $\exp (\mathbf{0})=I$ for any square zero matrix.

\section{B. Proof of Theorem 2}

Note that $\lambda_{i}(M)=\lambda_{i}\left(\exp \left(-S_{W}\right) \exp \left(S_{B}\right)\right), i=1,2, \ldots, d$. Denote $\widetilde{W}=\exp ^{1 / 2}\left(-S_{W}\right)$, we have from the Courant-Fischer minimax theorem that [14]

$$
\begin{aligned}
& \lambda_{i}\left(\exp \left(-S_{W}\right) \exp \left(S_{B}\right)\right)=\lambda_{i}\left(\widetilde{W}^{T} \exp \left(S_{B}\right) \widetilde{W}\right) \\
= & \max _{\operatorname{dim}(S)=i} \min _{\mathbf{v} \in S,\|\mathbf{v}\|_{2}=1} \mathbf{v}^{T} \widetilde{W}^{T} \exp \left(S_{B}\right) \widetilde{W} \mathbf{v} \\
= & \max _{\operatorname{dim}(S)=i} \min _{\mathbf{v} \in S,\|\mathbf{v}\|_{2}=1} \frac{(\widetilde{W} \mathbf{v})^{T} \exp \left(S_{B}\right)(\widetilde{W} \mathbf{v})}{(\widetilde{W} \mathbf{v})^{T}(\widetilde{W} \mathbf{v})} \cdot \frac{\mathbf{v}^{T} \widetilde{W}^{T} \widetilde{W} \mathbf{v}}{\mathbf{v}^{T} \mathbf{v}} .
\end{aligned}
$$

Recall that the eigenvalues of $\exp \left(-S_{W}\right)$ and $\exp \left(S_{B}\right)$ are $e^{-\nu_{d}} \geq e^{-\nu_{d-1}} \geq \cdots \geq e^{-\nu_{1}}$ and $e^{\mu_{1}} \geq e^{\mu_{2}} \geq \cdots \geq e^{\mu_{d}}$, respectively. For any $\mathbf{v} \in \mathbb{R}^{d},\|\mathbf{v}\|_{2}=1$, it follows that

$$
e^{-\nu_{1}} \leq \frac{\mathbf{v}^{T} \widetilde{W}^{T} \widetilde{W} \mathbf{v}}{\mathbf{v}^{T} \mathbf{v}}=\frac{\mathbf{v}^{T} \exp \left(-S_{W}\right) \mathbf{v}}{\mathbf{v}^{T} \mathbf{v}} \leq e^{-\nu_{d}},
$$

and

$$
e^{\mu_{i}}=\max _{\operatorname{dim}(S)=i} \min _{\mathbf{v} \in S,\|\mathbf{v}\|_{2}=1} \frac{(\widetilde{W} \mathbf{v})^{T} \exp \left(S_{B}\right)(\widetilde{W} \mathbf{v})}{(\widetilde{W} \mathbf{v})^{T}(\widetilde{W} \mathbf{v})} .
$$

Therefore, we have from (6.1)-(6.3) that

$$
e^{\mu_{i}-\nu_{1}} \leq \lambda_{i}(M) \leq e^{\mu_{i}-\nu_{d}}, \quad i=1,2, \ldots, d .
$$


On the other hand, we have

$$
\lambda_{i}\left(\exp \left(-S_{W}\right) \exp \left(S_{B}\right)\right)=\lambda_{i}\left(\exp ^{1 / 2}\left(S_{B}\right) \exp \left(-S_{W}\right) \exp ^{1 / 2}\left(S_{B}\right)\right) .
$$

Using the same trick, we can prove that for $i=1,2, \ldots, d$,

$$
e^{\mu_{d}-\nu_{d-i+1}} \leq \lambda_{i}(M) \leq e^{\mu_{1}-\nu_{d-i+1}} .
$$

A combination of (6.4) and (6.5) yields (3.17).

\section{Proof of Theorem 6}

Note that $V=\widetilde{V} \widetilde{V}^{T} V+\left(I-\widetilde{V} \widetilde{V}^{T}\right) V$, and $\cos \angle(V, \widetilde{V})=\left\|V^{T} \widetilde{V}\right\|_{2}$. Thus,

$$
\begin{aligned}
d_{i j} & =\left\|V^{T}\left(\widehat{\mathbf{x}}_{i}-\widehat{\mathbf{y}}_{j}\right)\right\|_{2} \\
& =\left\|\left(V^{T} \widetilde{V}\right) \widetilde{V}^{T}\left(\widehat{\mathbf{x}}_{i}-\widehat{\mathbf{y}}_{j}\right)+V^{T}\left(I-\widetilde{V} \widetilde{V}^{T}\right)\left(\widehat{\mathbf{x}}_{i}-\widehat{\mathbf{y}}_{j}\right)\right\|_{2} \\
& \leq \widetilde{d}_{i j}\left\|V^{T} \widetilde{V}\right\|_{2}+\left\|V^{T}\left(I-\widetilde{V} \widetilde{V}^{T}\right)\right\|_{2}\left\|\widehat{\mathbf{x}}_{i}-\widehat{\mathbf{y}}_{j}\right\|_{2} \\
& =\widetilde{d}_{i j} \cos \angle(V, \widetilde{V})+\sin \angle(V, \widetilde{V})\left\|\widehat{\mathbf{x}}_{i}-\widehat{\mathbf{y}}_{j}\right\|_{2} \\
& \leq \widetilde{d}_{i j} \cos \angle(V, \widetilde{V})+2 \sin \angle(V, \widetilde{V}),
\end{aligned}
$$

where we used $\left\|\widehat{\mathbf{x}}_{i}-\widehat{\mathbf{y}}_{j}\right\|_{2} \leq\left\|\widehat{\mathbf{x}}_{i}\right\|_{2}+\left\|\widehat{\mathbf{y}}_{j}\right\|_{2} \leq 2$. Using the same trick, we can prove that

$$
\widetilde{d}_{i j} \leq d_{i j} \cos \angle(V, \widetilde{V})+2 \sin \angle(V, \widetilde{V}),
$$

a combination of the above two inequalities gives (3.23).

\section{REFERENCES}

[1] N. AHMED. Exponential discriminant regularization using nonnegative constraint and image descriptor, IEEE 9th International Conference on Emerging Technologies, pp.1-6, 2013.

[2] S. AN, W. LiU, S. VENKATESH, AND H. YAN. Unified formulation of linear discriminant analysis methods and optimal parameter selection, Pattern Recognition, 44: 307-319, 2011.

[3] Z. Bai, J. Demmel, J. Dongarra, A. Ruhe, And H. VAn der Vorst. Templates for the Solution of Algebraic Eigenvalue Problems: A Practical Guide, SIAM, Philadelphia, 2000.

[4] P. Belhumeur, J. Hespanha, And D. Kriegman. Eigenfaces vs Fisherface: recognition using class-specific linear projection, IEEE Transactions on Pattern Analysis and Machine Intelligence, 19: 711-720, 1997.

[5] H. Cevikalp, M. Neamtu, M. Wilkes, And A. Barkana. Discriminative common vectors for face recognition, IEEE Transactions on Pattern Analysis and Machine Intelligence 27: 4-13, 2005.

[6] L. Chen, H. Liao, M. Ko, J. Lin, AND G. YU. A new lda-based face recognition system which can solve the small-sample-size problem, Pattern Recognition, 33: 1713-1726, 2000.

[7] B. CIPRA. The best of the 20th century: editors name top 10 algorithms, SIAM News 33(4), 2000.

[8] T. COVER AND P. HART. Nearest neighbor pattern classification, IEEE Transactions on Information Theory, 13: 21-27, 1967.

[9] F. Dornaika AND A. BosAghZADEH. Exponential local discriminant embedding and its application to face recognition, IEEE Transactions on Systems, Man, and Cybernetics-Part B: Cybernetics, 43: 921-934, 2013.

[10] R. Duda, P. Hart, And D. Stork. Pattern Classification, 2nd ed. New York: Wiley, 2000.

[11] B. Everitt, S. LAndaU, M. LeEse, And D. StAhl. Miscellaneous Clustering Methods, in Cluster Analysis, 5th Edition, John Wiley \& Sons, Ltd, Chichester, UK, 2011.

[12] R. FISHER. The statistical utilization of multiple measurements, Annals and Eugnenics, 8: 376-386, 1938.

[13] J. Friedman. Regularized discriminant analysis, Journal of the America Statistical Association, 84: 165-175, 1989.

[14] G.H. Golub And C.F. Van Loan. Matrix Computations. The Johns Hopkins university Press, 4th edtion, 2013.

[15] N.J. Higham. Functions of Matrices: Theory and Computation, SIAM, Philadelphia, 2008.

[16] P. HOWLAND AND H. PARK. Generalizing discriminant analysis using the generalized singular value decomposition, IEEE Transactions on Pattern Analysis and Machine Intelligence, 26: 995-1006, 2004.

[17] Y. JiA, F. NiE, AND C. Zhang. Trace ratio problem revisited, IEEE Transactions on Neural Networks, 20: 729-735, 2009.

[18] Z. JiA AND G.W. STEWART. An analysis of the Rayleigh-Ritz method for approximating eigenspaces, Mathematics of Computation, 70: 637-647, 2001. 
[19] G. Lu, Yong Wang. Feature extraction using a fast null space based linear discriminant analysis algorithm, Information Sciences, 193: 72-80, 2012.

[20] A. MARTINEZ. Recognizing imprecisely localized, partially occluded, and expression variant faces from a single sample perclass, IEEE Transactions on Pattern Analysis and Machine Intelligence, 24: 748-763, 2002.

[21] C. MOLER AND C.F. VAN LOAN. Nineteen dubious ways to compute the exponential of a matrix, twenty-five years later, SIAM Review, 45: 3-49, 2003.

[22] F. Nie, S. Xiang, Y. LiU, C. Hou, AND C. Zhang. Orthogonal vs. uncorrelated least squares discriminant analysis for feature extraction, Pattern Recognition Letters, 33: 485-491, 2012.

[23] F. Nie, S. XIANg, Y. Song, AND C. Zhang. Extracting the optimal dimensionality for local tensor discriminant analysis, Pattern Recognition, 42: 105-114, 2009.

[24] F. Nie, S. XIANG, Y. Song AND C. Zhang. Orthogonal locality minimizing globality maximizing projections for feature extraction, Optical Engineering, 18, Issue 1, 2009.

[25] F. Nie, S. XiAng, Y. JiA, C. ZhAng, And S. YAn. Trace ratio criterion for feature selection. Proceedings of the 23rd AAAI Conference on Artificial Intelligence, pp. 671-676, 2008.

[26] F. Nie, S. XIAng, And C. Zhang. Neighborhood minmax projections, The TwentiethInternational Joint Conference on Artificial Intelligence (IJCAI), Hyderabad, India, pp. 993-997, 2007.

[27] F. Nie, S. XiAng, Y. Song, AND C. ZHAng. Optimal dimensionality discriminant analysis and its application to image recognition. 1st Workshop on Component Analysis Methods for Classification, Clustering, Modeling and Estimation Problems in Computer Vision (CVPR workshop), 2007.

[28] C. PARK AND H. PARK. A comparision of generalized linear discriminant analysis algorithms, Pattern Recognition, 41: 1083-1097, 2008.

[29] P. Phillips, H. Moon, P. J. Rauss, And S. RizVI. The FERET evaluation methodology for face recognition algorithms, IEEE Transactions on Pattern Analysis and Machine Intelligence, 22: 1090-1104, 2000.

[30] M. Popolizio And V. Simoncini. Acceleration techniques for approximating the matrix exponential operator, SIAM Journal on Matrix Analysis and Aplications, 30: 657-683, 2008.

[31] Y. SAAD. Numerical Methods for Large Eigenvalues Problems, 2nd edition, SIAM, Philadelphia, 2011.

[32] Y. SAAD. Analysis of some Krylov subspace approximations to the matrix exponential operator, SIAM Journal on Numerical Analysis, 29: 209-228, 1992.

[33] V. Simoncini AND D. SzYLD. Theory of inexact Krylov subspace methods and applications to scientific computing, SIAM Journal on Scientific Computing, 25: 454-477, 2003.

[34] G. SLEIJPEN AND H. VAN DER VORST. A Jacobi-Davidson iteration method for linear eigenvalue problems, SIAM Journal on Matrix Analysis and Aplications, 17: 401-425, 1996.

[35] D. SORENSEN. Implicit application of polynomial filters in a k-step Arnoldi method, SIAM Journal on Matrix Analysis and Aplications, 13: 357-385, 1992.

[36] G.W. STEWART. Matrix Algorithms II: Eigensystems, SIAM, Philadelphia, 2001.

[37] M. Turk And A. Pentland. Eigenfaces for recognition, Cognitive Neuroscience, 3: 71-86, 1991.

[38] S.G. WANG, M. Wu, AND Z.Z. JiA. Matrix inequalities (in Chinese), Science Academic Press, Beijing, 2 nd edition, 2005.

[39] S. WAng, H. Chen, X. Peng, AND C. ZHOU. Exponential locality preserving projections for small sample size problem, Neurocomputing, 74: 36-54, 2011.

[40] S. WANG, S. YAN, J. YANG, C. ZHoU, AND X. FU. A general exponential framework for dimensionality reduction, IEEE Transactions on Image Processing, 23: 920-930, 2014.

[41] X. WANG AND X. TANG. A unified framework for subspace face recognition, IEEE Transactions on Pattern Analysis and Machine Intelligence, 26: 1222-1228, 2004.

[42] G. WU AND Y. WEI. On analysis of projection methods for rational function approximation to the matrix exponential, SIAM Journal on Numerical Analysis, 48: 191-197, 2010.

[43] G. WU, W. XU, AND H. LENG. Inexact and incremental bilinear Lanczos components algorithms for high dimensionality reduction and image reconstruction, Pattern Recognition, 48: 244-263, 2015.

[44] G. Wu, H. PANG, AND J. SUn. Preconditioning the restarted and shifted block FOM algorithm for matrix exponential computation, arXiv preprint, available online at http://arxiv.org/abs/1405.0707.

[45] L. YAN AND J. PAN. Two-dimensional exponential discriminant analysis and its application to face recognition, International Conference on Computational Aspects of Social Networks (CASoN), pp. 528-531, 2010.

[46] J. YE. Charaterization of a family of algorithms for generalized discriminant analysis on undersampled problems, Journal of Machine Learning Research, 6: 483-502, 2006.

[47] J. Ye, R. JANARDAn, C.H. PARK, AND H. PARK. An optimization criterion for generalized discriminant analysis on undersampled problems, IEEE Transactions on Pattern Analysis and Machine Intelligence, 26: 982-994, 2004.

[48] J. YE AND Q. LI. A two-stage linear discriminant analysis via QR decomposition, IEEE Transactions on Pattern Analysis and Machine Intelligence, 27: 929-941, 2005.

[49] H. YU AND H. YANG. A direct LDA algorithm for high-dimensional data with application to face recognition, Pattern Recognition, 34: 2067-2070, 2001. 
[50] T. Zhang, B. FAng, Y. TAng, Z. Shang, AND B. XU. Generalized discriminant analysis: a matrix exponential approach, IEEE Transactions on Systems Man and Cyberrnetics-Part B: cyberrnetics, 40: 186-197, 2010.
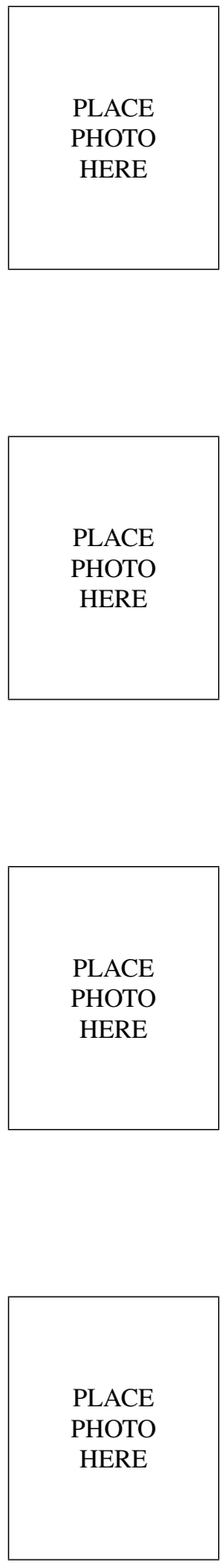

Li-jia Zhang received the B.S.degree from Department of Mathematics, Yancheng Normal University, in 2010, M.S.degree from School of Mathematics and Statistics, Jiangsu Normal University, in 2013. His current research mainly focuses on large sparse matrix computations and pattern recognition.
Gang Wu is a Professor of Department of Mathematics, School of Science, China University of Mining and Technology. He received the B.S.degree from School of Mathematics, Shandong University, in 1998, M.S.degree from Department of Applied Mathematics, Dalian University of Technology, in 2001, and Ph.D. degree from Institute of Mathematics, Fudan University, in 2004. His current research mainly focuses on large sparse matrix computations, pattern recognition and data mining.

Ting-ting Feng received the B.S.degree from Department of Mathematics, Xingtan College of Qufu Normal University, in 2012, M.S.degree from School of Mathematics and Statistics, Jiangsu Normal University, in 2015. Currently, she is a PhD student in Department of Mathematics, East China Normal University. Her current research mainly focuses on large sparse matrix computations and numerical linear algebra with applications.
Meng Yang received the B.S.degree from Department of Mathematics, Xingtan College of Qufu Normal University, in 2012, M.S.degree from School of Computer Science and Technology, Soochow University, in 2015. His current research mainly focuses on pattern recognition and image processing. 